\title{
Review
}

Wei Gao ${ }^{a}$, Zhaoqiang Zheng ${ }^{\mathrm{a}}$, Peiting Wen, Nengjie Huo* and Jingbo Li*

\section{Novel two-dimensional monoelemental and ternary materials: growth, physics and application}

https://doi.org/10.1515/nanoph-2019-0557

Received December 28, 2019; revised February 2, 2020; accepted February 2, 2020

\begin{abstract}
Two-dimensional (2D) materials have undergone a rapid development toward real applications since the discovery of graphene. At first, graphene is a star material because of the ultrahigh mobility and novel physics, but it always suffered from zero bandgap and limited device application. Then, 2D binary compounds such as transition-metal chalcogenides emerged as complementary materials for graphene due to their sizable bandgap and moderate electrical properties. Recently, research interests have turned to monoelemental and ternary 2D materials. Among them, monoelemental 2D materials such as arsenic (As), antimony (Sb), bismuth (Bi), tellurium (Te), etc., have been the focus. For example, bismuthene can act as a 2D topological insulator with nontrivial topological edge states and high bulk gap, providing the novel platforms to realize the quantum spin-Hall systems. Meanwhile, ternary $2 \mathrm{D}$ materials such as $\mathrm{Bi}_{2} \mathrm{O}_{2} \mathrm{Se}, \mathrm{BiOX}$ and $\mathrm{CrOX}(\mathrm{X}=\mathrm{Cl}, \mathrm{Br}, \mathrm{I})$ have also emerged as promising candidates in optoelectronics and spintronics due to their extraordinary mobility, favorable band structures and intrinsic ferromagnetism with high Curie temperature. In this review, we will discuss the recent works and future prospects on the emerging monoelemental and ternary materials in terms of their structure, growth, physics and device applications.
\end{abstract}

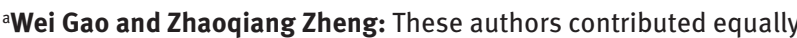
to this work.

*Corresponding authors: Nengjie Huo and Jingbo Li, Institute of Semiconductors, South China Normal University, Guangzhou 510631, P.R. China, e-mail: njhuo@semi.ac.cn (N. Huo); jbli@m.scnu.edu.cn (J. Li).

Wei Gao and Peiting Wen: Institute of Semiconductors, South China Normal University, Guangzhou 510631, P.R. China. https://orcid. org/0000-0002-6491-6128 (W. Gao)

Zhaoqiang Zheng: School of Materials and Energy, Guangdong University of Technology, Guangzhou 510006, P.R. China
Keywords: two-dimensional monoelemental materials; ternary materials; bandgap; optoelectronics; ferromagnetism.

\section{Introduction}

Through fast development in 15 years, two-dimensional layered materials (2DLMs) such as graphene $(\mathrm{Gr})(0 \mathrm{eV})$ have not only become a revolutionary material but also received large-scale high-quality production [1-4]. Recently, graphene-based magic-angle superconductor [5], hybridization filter membrane [6], flexible electronics $[7,8]$ and transfer technology have been achieved $[9,10]$, which brings a new groundbreaking period. Recently, a new cousin of graphene, called "plumbene," which has the famous Weaire-Phelan bubble structure, has been considered to bring a new boom in the post-Moore law period [11]. Since 2014, similar with Gr, composed of a single element with an atomic thickness profile, black phosphorus (BP), as a star monoelemental material with a tunable bandgap (0.3-2.0 eV) [12], low grain boundary and high carrier mobility $\left(10^{3} \mathrm{~cm}^{2} \cdot \mathrm{V}^{-1} \cdot \mathrm{s}^{-1}\right)$ [13], has been applied in anisotropic lithium and sodium intercalation [14], (opto) electronic devices [15-17], sensors, medical treatment and energy [18]. However, excellent BP devices have to be covered or sandwiched by hexagonal-nitrile boron (h-BN) due to their poor air stability [19]. Meanwhile, extensive studies on alloy engineering (such as black-arsenic phosphorus [B-AsP]) [20, 21], allotrope synthesis (Violet phosphorus) [22] and monoelemental doping (selenium doping-black phosphorus) [23] have been carried out to resolve the problem of high chemical activity exposure to air and to improve optoelectronic applications. Most importantly, more and more predicted stable monoelemental materials with novel physical properties have been experimentally synthesized and studied in recent years.

In addition, due to the quantum confinement effect and interlayer coupling behavior, transition or post transition metal chalcogenide (transition/post transition metal chalcognides [TMCs/PTMCs]) nanosheets such as MX or $\mathrm{MX}_{2}(\mathrm{M}=\mathrm{W}, \mathrm{Mo}, \mathrm{V}, \mathrm{Re}, \mathrm{Pd}, \mathrm{Pt}, \mathrm{Sn}, \mathrm{In} ; \mathrm{X}=\mathrm{S}$, Se, Te $)$ are the 
most interesting fields for semimetal or semiconductorbased 2DLMs. Their bandgap is in the range of $0.2-2.1 \mathrm{eV}$, with broadband optical absorption efficient $\left(>10^{4} \mathrm{~cm}^{-1}\right)$ and moderate carrier mobility $\left(10-10^{2} \mathrm{~cm}^{2} \cdot \mathrm{V}^{-1} \cdot \mathrm{s}^{-1}\right)$. Moreover, crystal type, bandgap structure, unique physics, (opto)electronic devices performance and biomedical application have been discussed by a number of reviews and research papers in recent years [23-37]. Nevertheless, because of the covalent bonding between the anion/ion atoms and the general weak interlayer coupling effect, TMCs and their heterostructures have suffered from intrinsic/extrinsic disorder (vacancies, anti-site, substitution) and interface impurity (small molecules), leading to the downtrend or strange phenomenon in electronic and optoelectronic properties [38, 39]. Fortunately, the charge transport [40], photoluminescence [41], layer-dependent magnetism [42] and electrical properties [43-46] of TMCs can be enhanced via some specific kinds of defects (point substitution or deep levels). Among them, monoelement doping in 2DLMs has attracted researchers because of the unique properties of monoelements. Therefore, novel monoelemental and ternary two-dimensional (2D) materials with lower disorder and dramatic physics such as topological insulator (TI) behavior and quantum spin-orbit effect are being studied in the recent 3 years.

So far, among the 2D layered materials, five kinds of hot monoelemental 2D materials, including arsenic (As), antimony ( $\mathrm{Sb})$, tellurium (Te), bismuth (Bi) and selenium (Se) (belonging to $\mathrm{V}_{\mathrm{A}}-\mathrm{VI}_{\mathrm{A}}$ group), have been successfully synthesized and discovered with various crystal phases $[47,48]$ instead of 2D mental oxide [49] after the discovery of BP. Most importantly, the growth, physics and application of those materials in monolayer or few/multilayered forms are distinctly different from the binary 2DLMs. For instance, nanowires, nanosheets and bulk structure can be found by different researchers in quasi-2D Te. Meanwhile, the carrier mobilities of 2D Te and Sb nanosheets were found to be as high as $10^{2}-10^{3} \mathrm{~cm}^{2} \cdot \mathrm{V}^{-1} \cdot \mathrm{s}^{-1}$, with an excellent on/off ratio $\left(\sim 10^{6}\right)$ [48]. Thus, these new monoelemental materials and their heterostructures can hold great promise in next-generation nanodevices. However, their large-area synthesis, air stability, photo-response and biomedical application remain critical issues to be addressed. Moreover, instead of ternary alloy 2D materials MXY and $\mathrm{M}_{1} \mathrm{M}_{2} \mathrm{X}$, such as $\operatorname{ReS}_{2 \mathrm{x}} \mathrm{Se}_{2(1-\mathrm{x})}$ and $\mathrm{Mo}_{\mathrm{x}} \mathrm{W}_{1-\mathrm{x}} \mathrm{S}_{2}$ $[50,51]$, van der Waals (vdWs) MOX nanosheets $(\mathrm{M}=\mathrm{Bi}$, $\mathrm{Fe}, \mathrm{Co}, \mathrm{Mn}, \mathrm{Cr}$, lanthanide or actimide metals; $\mathrm{X}=\mathrm{F}, \mathrm{Cl}$, $\mathrm{Br}$, I) were theoretically predicted and experimentally achieved for the discovery of intrinsic ferromagnetic and spin electronic [52]. For example, ferromagnetic CrOX $(\mathrm{X}=\mathrm{Cl}$ or $\mathrm{Br})$ delivered a Curie temperature up to 160 and
$129 \mathrm{~K}$ [53]. But very rare experimental works of MOX have been performed. However, the corresponding synthesis, novel structures and other challenges should be improved and overcome. Thus, it is indispensable to focus on these novel materials to accelerate the synthesis process and the development of magnetic and spintronic applications. Among them, air-stable $\mathrm{Bi}_{2} \mathrm{O}_{2}$ Se nanosheets with an ultralow electron effective mass $\left(0.14 \mathrm{~m}_{0}\right)$ and a narrow bandgap $(\sim 0.8 \mathrm{eV})$ have exhibited extraordinary electron mobility $\left(28,900 \mathrm{~cm}^{2} \cdot \mathrm{V}^{-1} \cdot \mathrm{s}^{-1}\right.$ at $\left.2 \mathrm{~K}\right)$ and high on/off ratio $\left(10^{6}\right)$ [54]. In contrast, the layer-by-layer interaction is electrostatic force instead of vdWs. A number of related articles about photodiodes, photodetectors (PDs) and field-effect transistors (FETs) are reported. Very recently, research interests have turned to monoelemental and ternary 2D materials. However, a review about these new emerging materials has been rarely reported. As novel and promising 2D materials, they have some common points in terms of their physical properties and applications, and it is necessary to discuss them together in order to highlight their novel characteristics and challenges. In this review, first, we will focus on the structure, synthesis and physics of five kinds of hot monoelemental 2D materials (As, Sb, $\mathrm{Bi}, \mathrm{Te}$ and Se). Second, we will also discuss some novel ternary 2D materials, including $\mathrm{Bi}_{2} \mathrm{O}_{2} \mathrm{Se}$, $\mathrm{BiOX}$ and $\mathrm{CrOX}$ ferromagnetic materials to date. Importantly, their applications in electronics, optoelectronics, vapor sensors, spintronics, biomedicine and so on are also explored for each of the above 2D materials. Lastly, we will figure out some formidable challenges and discuss the expectation for those 2D materials.

\section{Elementary materials}

The 2D BP nanosheets have been demonstrated to exhibit unique physical and optoelectronic properties including the quantum Hall effect, saturable absorption and high performance of transistors and infrared PDs [55-57]. Subsequently, focus has been on the monolayer and fewlayer monoelemental 2D materials of group-VA elements (As, Sb, Bi, Te and Se), showing the promising candidates in future applications in electrics and optics [58].

\subsection{Arsenic}

As exhibits different crystal orientations, which are dependent on the bulk composition. Herein, there are two stable phases existing in As to be discussed in this review. Generally, orthorhombic As ( $\varepsilon$-As or b-As) is consistent 
with the cousin of $\mathrm{BP}$ and is abundant in natural shining luster mineral $[59,60]$. Reasonably, in the case of alloy engineering, air-stable and low toxic B-AsP nanosheets with a narrow bandgap of $0.2-0.3 \mathrm{eV}$ in bulk were prepared and used for FET and PD applications [20, 61]. As seen in Figure 1A, puckered six-membered rings of As atoms (belongs to Pmna. space group) stack layer by layer, which is in agreement with graphene sheets in graphite. Gray As is $\beta$-As with a buckled honeycomb structure (see Figure 1B), and the corresponding characterization has been reported first in 2001 [68]. It is convincing that gray As is the most stable form of arsenic in the rhombohedral space group of $R 3 m[69,70]$. Moreover, a mixed single crystal of $\varepsilon$-As combined with $\beta$-As was found in natural pararsenolamprite from Japan [70]. Those mixed crystals can be also obtained in the exact growth temperature range.

Nowadays, mechanical exfoliation (ME) [62, 67], liquid-phase exfoliation (LPE) [64, 71-73] and vdWs epitaxial growth [65] are mainly used to obtain 2D black/ gray arsenic and arsenene. In 2018, Chen et al. and Zhong et al. fabricated Hall-bar devices and back-gated monolayer b-As FETs by using the well-known scotch-tape method, respectively. The thickness of the monolayer

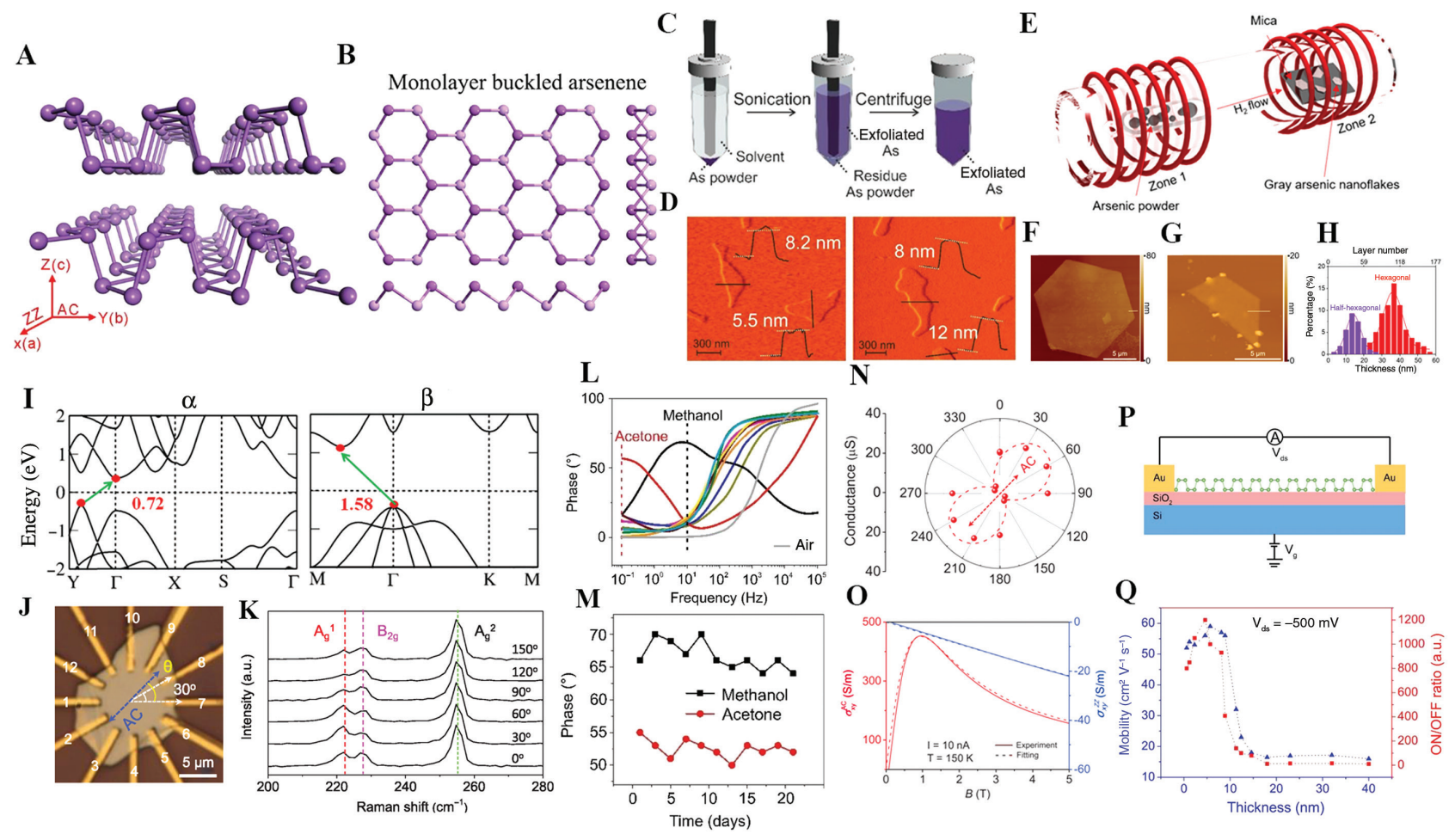

Figure 1: Structure, growth, physics and application of 2D As.

(A) Layered crystal structure of puckered b-As. Reproduced with permission from Ref. [62]. Copyright 2018, Wiley-VCH. (B) geometric structure of monolayer buckled arsenene. Reproduced with permission from Ref. [63]. Copyright 2019, Elsevier Ltd. (C) Schematic of the tip ultrasonication setup with minimizing exposure to ambient air. (D) AFM topography of arsenene showing several few-layered arsenene flakes with terraces of different heights as obtained from AFM images. (C, D) Reproduced with permission from Ref. [64]. Copyright 2019, Wiley$\mathrm{VCH}$. (E) Schematic illustration of a two-zone tube furnace configuration for the growth of gray arsenic nanoflakes. (F, G) AFM images and corresponding height profiles of hexagonal and half-hexagonal gray arsenic flakes. $(\mathrm{H})$ Statistical diagram of the thicknesses of gray arsenic nanoflakes. (E-H) Reproduced with permission from Ref. [65]. Copyright 2019, American Chemical Society. (I) Electronic band structure of various monolayer allotropes of As calculated using the GGA-PBE method. The Fermi level is set to zero. Reproduced with permission from Ref. [66]. Copyright 2018, Royal Society of Chemistry. (J) Optical image of a 12-terminal device used for Raman measurement. The b-As thickness is $132 \mathrm{~nm}$. (K) Representative Raman spectra of the multilayer b-As with $A_{\mathrm{g}}^{1}$, $B_{2 g}$ and $A_{g}^{2}$ modes under excitation with laser polarized along different directions. (J, K) Reproduced with permission from Ref. [67]. Copyright 2018, Wiley-VCH. (L) Vapor senor based on arsenene nanosheets: impedance phase spectra in the presence of either methanol, acetone and ambient air. (M) The durability of the methanol and acetone vapor sensors for 3 weeks. (L, M) Reproduced with permission from Ref. [64]. Copyright 2018, Wiley-VCH. (N) Angular-dependent electrical conductance of multilayer b-As, showing maximum along the AC direction. (0) Magnetic field-dependent transverse conductivity $\sigma_{x y}$ of b-As along AC and ZZ directions at 150 K. (N, O) Reproduced with permission from Ref. [67]. Copyright 2018, Wiley-VCH. (P) Cross-section of a monolayer b-As FET. (Q) Carrier mobility and on/off ratios as a function of the thicknesses for b-s under the $V_{d s}$ of -0.5 V. (P, Q) Reproduced with permission from Ref. [62]. Copyright 2018, Wiley-VCH. 
sample exfoliated from natural mineral is in the range of $0.6-0.7 \mathrm{~nm}[62,67]$. After exposure to air for a long time, b-As was oxidized and showed a weak performance in electronics. Prominently, in recent years, LPE with a sealed environment by ultrasonic/shear force treatment and high-speed centrifugation has been the mainstream method used to prepare 2D arsenic and arsenene (see Figure $1 \mathrm{C}$ and D). Sturala et al. fabricated chloromethylene $(-\mathrm{CHCl})$ functionalized few-layered arsenic in dry dimethylformamide during the shear-force exfoliation. The as-prepared sample showed enhanced luminescence with a broad maximum around $535 \mathrm{~nm}$ [71]. To overcome the oxidation of BP and arsenic, Sturala and coworkers further adopted covalent and noncovalent modification during the liquid exfoliation process. Meanwhile, the modifications can not only stabilize the nanostructures but also tune the bandgap and make an unimaginable effect on properties [74]. Moreover, large-scale arsenic nanosheets $(>10 \mu \mathrm{m})$ are needed to test the electronic, sensor and optoelectronic devices. In 2019, layered gray As nanoflakes on mica substrates were firstly successfully synthesized via a vdWs epitaxial method by Hu et al. [65] (see Figure 1E-H). It should be noted that gray As crystals can be obtained at a crystallization temperature above $300^{\circ} \mathrm{C}$. In contrast, a mixture of b-As and gray As will be easily formed in the range of $200-300^{\circ} \mathrm{C}$. Gray As nanosheets can further grow thinner to several nanometers by improving the crystalline condition. Interestingly, a polyethylene glycol (PEG) inert coating can protect gray As nanoflakes from being oxidized in air because of the weakest interfacial interaction with an average $E_{\text {int }}$ of -1.51 $\mathrm{eV}$ [65]. In the future, chemical vapor deposition (CVD) and molecular beam epitaxy (MBE) are two alternative ways to prepare few-layered gray arsenic. However, due to the metastability of b-As, the synthesis process has to be operated in an inert atmosphere in whole period.

$2 \mathrm{D}$ arsenic/arsenene is unstable in $\varepsilon$-form but air-stable in $\beta$-form because of the minimum energy configuration. Chen et al. found that $\beta$-As nanotubes were stable at room temperature and as stiff as silicon by means of the density functional theory (DFT) [63]. 2D $\varepsilon$-arsenene and $\beta$-arsenene can exhibit a direct bandgap semiconductor of $2.47 \mathrm{eV}$ and $2.49 \mathrm{eV}$, respectively [75]. Based on hybrid functional results (the equation of GW-Bethe-Salpeter), the calculated optical bandgap of arsenene is only $1.6 \mathrm{eV}$ because of the significant exciton effect, whereas fewlayered or multilayered $\varepsilon$-arsenic and $\beta$-arsenic deliver an indirect bandgap down to $0.72 \mathrm{eV}$ and $1.58 \mathrm{eV}$ by HSE06 level theoretical calculation, respectively (see Figure 1I) [66]. It is well known that the band structure of 2DLMs is subjected to mechanical strain, including compression and tensile strain. For instance, under a biaxial tensile strain of $4 \%$ and $11.7 \%$, the band structure of $\beta$-arsenene can change to direct bandgap and 2D TI, respectively [76]. To date, anisotropy $\varepsilon$-As and $\beta$-As nanosheets with low symmetries have been attractive because of the potential application in dichroism, photodetection, polarization imaging and other novel devices $[77,78]$. According to the difference between in-plane and out-of-plane interatomic distances, the anisotropy of $\beta$-As is $25 \%$ [79]. Intuitively, angle-resolved Raman spectrum is used to investigate the anisotropy phonon vibrational physics of 2DLMs [80]. Liu et al. were the first to study the anisotropic Raman spectrum of $\mathrm{b}-\mathrm{As}_{0.83} \mathrm{P}_{0.17}$ [81], and Chen et al. systematically figured out that Raman peaks of $223.6 \mathrm{~cm}^{-1}$ (out-of-plane, $\mathrm{A}_{\mathrm{g}}^{1}$ ), $230.2 \mathrm{~cm}^{-1}$ (in-plane, $\mathrm{B}_{2 \mathrm{~g}}$ ) and $257.9 \mathrm{~cm}^{-1}$ (in-plane, $\mathrm{A}_{\mathrm{g}}^{2}$ ) of pure b-As changed in a period along the armchair (AC) and zigzag (ZZ) directions (see Figure 1J and K) [67]. Moreover, this Raman result is consistent with anisotropic electrical behaviors and thermal transport. For the former, the conductance of b-As along the AC direction is 6.4 times larger than that along the $\mathrm{ZZ}$ direction. For the latter, the thermal conductivity $(\kappa)$ anisotropic ratio reached 1.6 at room temperature. Nevertheless, there have been no reports of experiments on the anisotropic behaviors for $\beta$-As nanosheets. In electrical physics, it is theoretically predicted that the hole mobility of bulk b-As and $\beta$-As can exceed $10^{3} \mathrm{~cm}^{2} \cdot \mathrm{V}^{-1} \cdot \mathrm{s}^{-1}[82,83]$. However, the experimental results are much lower than the theoretical value, which is related with the defects in b-As crystals and the corresponding dielectric environments. Nowadays, the maximum experimental hole mobility of few-layered b-As nanosheets with a thickness of around $6 \mathrm{~nm}$ has reached $\sim 59 \mathrm{~cm}^{2} \cdot \mathrm{V}^{-1} \cdot \mathrm{s}^{-1}$. Moreover, Wang et al. theoretically found that monolayer arsenene showed a low electron/hole carrier mobility of $21 / 66 \mathrm{~cm}^{2} \cdot \mathrm{V}^{-1} \cdot \mathrm{s}^{-1}$ at room temperature based on the phonon-electron interaction strength [63].

In recent years, due to their moderate optical bandgap and strong interaction with small molecules, arsenic/arsenene nanosheet and its heterostructure have been predicted to be promising candidates for FETs and solvent vapor sensors. Some theoretical models describing arsenic/arsenene in promising applications based on accurate $a b$ initio methods are helpful in constructing the next-generation 2DLM nanodevices. In general, gray As is the most ideal and stable form to be applied in nanodevices. In 2017, Liu et al. theoretically studied the absorption energy of $\mathrm{CO}, \mathrm{CO}_{2}, \mathrm{~N}_{2}, \mathrm{NH}_{3}, \mathrm{H}_{2} \mathrm{O}, \mathrm{NO}$ and $\mathrm{NO}_{2}$ on monolayer gray arsenene without considering the effect of defects. Their results show that NO and 
$\mathrm{NO}_{2}$ can lead to a magnetic moment of $1 \mu \mathrm{B}$ in arsenene [84], which is due to the spin polarization in the system induced by the adsorbed $\mathrm{NO}_{2}$ molecules. In 2019, BeladiMousavi et al. fabricated a few-layered gray As vapor sensor with an interdigitated gold electrode. The exfoliated As nanosheet can selectively detect either methanol or acetone vapor depending on concrete frequency during a period of 3 weeks (see Figure 1L and M) [64]. Moreover, temperature, thickness and polarized direction also play important roles in the electrical properties of arsenic nanosheets, which act as a conductive constituent to achieve suitable band alignment in nano-electronics devices. The calculated electrical conductivity of multilayered gray As is as large as $3.5 \times 10^{3} \mathrm{~S} \cdot \mathrm{m}^{-1}$, without gate effect (on/off ratio is $\sim 1$ ). It is attributed to the overlap of the energy levels for semimetal As [65]. In contrast, a multilayered b-As Hall-bar device with a narrow bandgap of $0.3 \mathrm{eV}$ revealed the highest electrical conductance along the $\mathrm{AC}$ direction (see Figure $1 \mathrm{~N}$ ). The anisotropic ratio in mobility can approximately reach 175 , which is much higher than that of other in-plane anisotropic materials (see Figure 10) [85]. Moreover, few-layered b-As FETs showed a high on/off ratio of $\sim 1.2 \times 10^{3}$ and a moderate hole mobility of $59 \mathrm{~cm}^{2} \cdot \mathrm{V}^{-1} \cdot \mathrm{s}^{-1}$ (see Figure $1 \mathrm{P}$ and Q) [62]. Those values can be further enhanced by improving the device configuration such as $\mathrm{h}$-BN encapsulation and polymethyl methacrylate (PMMA) passivation. Depending on the excellent electrical properties of b-AsP and its heterostructure, the pure b-As or gray As-based photodiode and PDs will exhibit a tremendous performance in the future $[61,81]$.

\subsection{Antimony}

Antimonene is defined as a monolayer of $\mathrm{Sb}$ atoms, which has attracted great attention because of their strong spin-orbit coupling (SOC) and a drastic evolution of electric structures with varying thicknesses [86]. In contrast to puckered armchair-like phosphorene, the most stable antimonene holds a buckled honeycomb structure with a much stronger spin-orbital coupling. Zhang et al. studied the band structures of monolayer antimonene in its hexagonal $\beta$ phase through DFT calculations and showed an indirect bandgap of $2.28 \mathrm{eV}$ [87]. The $\beta$-Sb has a hexagonal lattice with the buckled surface as shown in Figure 2A. It was reported that the bond length between neighboring Sb atoms is $2.84 \AA$ and the bond angle is $89.9^{\circ}$ [88]. Compared to widely reported phosphorene, antimonene possesses robust environmental stability, adaptive for long-term applications [94].
On the other hand, antimonene is an indirect bandgap semiconductor, which can lead to a lower energy conversion, thus restricting its application in photonic devices [75]. So far, many strategies have been developed to prepare monolayer and few-layer antimonene, including the traditional micromechanical exfoliation, liquid phase exfoliation and epitaxial growth, etc. In 2016, Ares et al. prepared a highly stable antimonene by ME with thickness as thin as $\sim 0.9 \mathrm{~nm}$ and predicted an electronic gap of $\approx 1 \mathrm{eV}$, which is very promising for optoelectronics applications [94]. Through the LPE method, several groups have achieved a few layers of antimonene with a thickness below $5 \mathrm{~nm}$ [95-97]. Recently, Peng et al. successfully obtained few-layer hexagonal and functionalized antimonene nanosheets from $\mathrm{SbCl}_{3}$ solutions for the first time by exclusively promoting their anisotropic growth in a colloidal solution where oleylamine was selected as the reducing agent [89]. Figure 2B shows the transmission electron microscope (TEM) and highresolution TEM (HRTEM) images of the as-prepared hexagonal antimonene nanosheets with a lateral size of $0.5-1.5 \mu \mathrm{m}$ and thickness of $\sim 5 \mathrm{~nm}$. The HRTEM revealed a regular atomic arrangement and lattice fringes of hexagonal patterns along the (100) direction with a lattice spacing of $0.24 \mathrm{~nm}$. The epitaxial growth method has also been used to grow antimonene on different substrates. Shao et al. reported the epitaxial growth of atomically thin flat honeycomb monolayer antimonene on Ag (111) substrate shown in Figure 2C [90]. Zhu et al. grew the Sb monolayer and $\mathrm{Sb}$ island on $\mathrm{Cu}$ (111) substrate shown in Figure 2D and demonstrated that the monolayer island can exhibit significantly robust $1 \mathrm{D}$ topological edge states above the Fermi level [91]. Ge (111) was also used as a substrate to grow 2D single- and few-layered antimonene by evaporating antimony crystals [98]. Ji et al. developed the vdWs epitaxy growth of few-layered antimonene monocrystalline polygons with high stability on mica and flexible substrate. The authors characterized the Raman spectra of antimonene as a function of thickness, as shown in Figure 2E [92]. The bulk Sb exhibits two typical Raman peaks located at around $111 \mathrm{~cm}^{-1}$ and $149 \mathrm{~cm}^{-1}$, which are assigned as in-plane $E_{\mathrm{g}}$ mode and out-of-plane $A_{1 g}$, respectively. Both $E_{g}$ and $A_{1 g}$ peak frequencies increase with the decreased thickness, which is probably attributed to the lattice constant shrinks. Last but not the least, Duan et al. used their famous two-step CVD method to fabricate a $\mathrm{Sb} / \mathrm{WSe}_{2}$ heterostructure. The thickness of $\mathrm{Sb}$ nanosheet on $\mathrm{WSe}_{2}$ can be effectively reduced to monolayer [45].

As for the application of antimonene in devices, few works has been reported. Pizzi et al. simulated the 

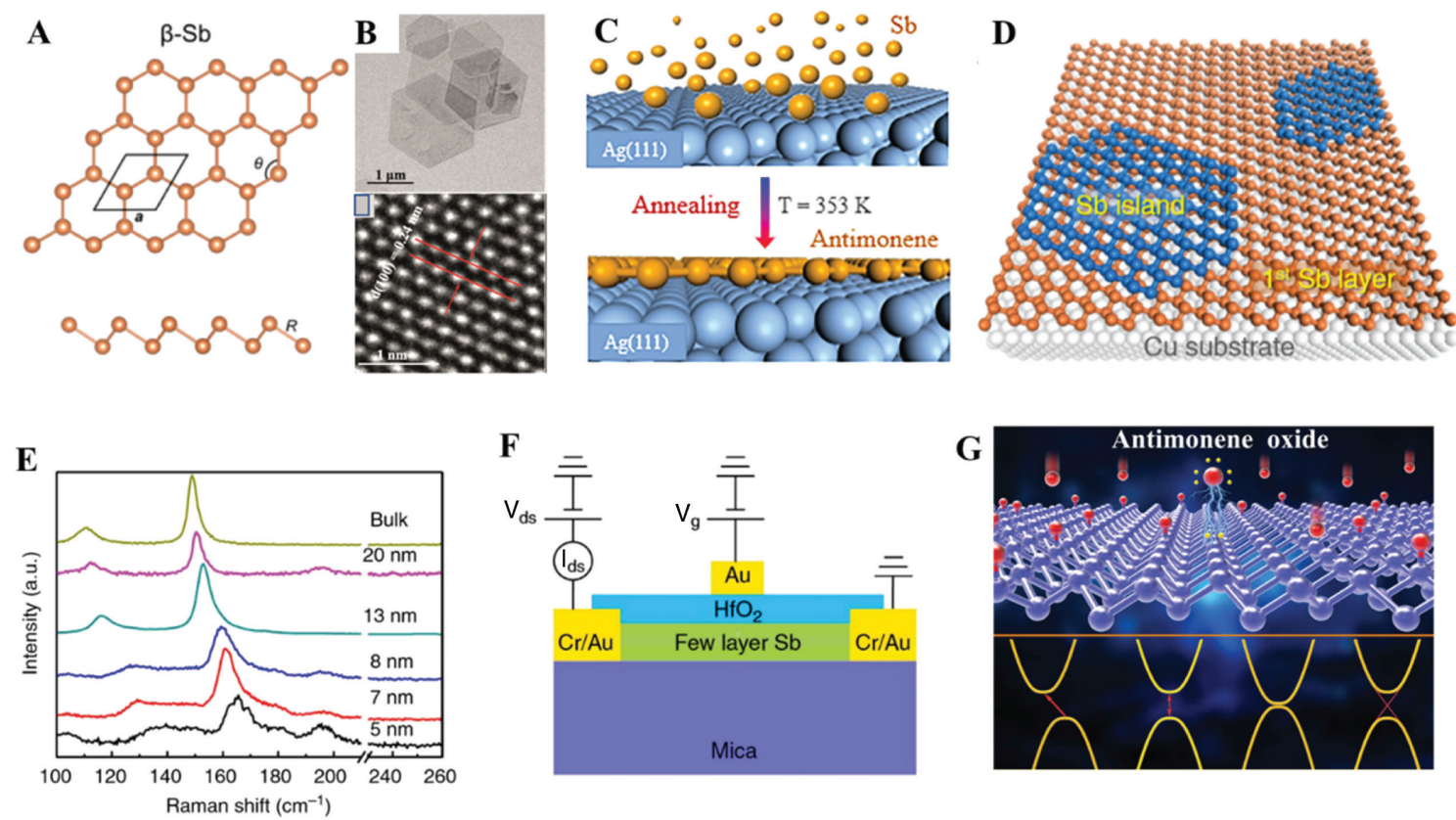

Figure 2: Structure, growth, physics and application of 2D Sb.

(A) Structural configurations of antimonene allotropes $\beta$-Sb. Reproduced with permission from Ref. [88]. Copyright 2019, American Chemical Society. (B) TEM and HRTEM images of hexagonal antimonene nanosheets with a lattice spacing of $0.24 \mathrm{~nm}$. Reproduced with permission from Ref. [89]. Copyright 2019, Wiley-VCH. (C) Schematic of fabrication process of antimonene on Ag (111) substrate. Reproduced with permission from Ref. [90]. Copyright 2018, American Chemical Society. (D) Structural model of the buckled antimonene monolayer (orange) and antimonene nanoisland (blue) on Cu (111) substrate. Reproduced with permission from Ref. [91]. Copyright 2019, American Chemical Society. (E) Raman spectra of antimonene polygons with thicknesses varying from $5 \mathrm{~nm}$ to bulk. (F) Schematic illustration of top-gated antimonene thin film transistors. (E, F) Reproduced with permission from Ref. [92] Copyright 2016, Springer Nature. (G) Sketch map of antimonene oxide. The bottom panel represents the electronic band structure of antimonene oxides with the different oxygen concentration. Reproduced with permission from Ref. [93]. Copyright 2017, American Chemical Society.

performances of double-gate metal-oxide-semiconductor FETs based on arsenene and antimonene monolayers via first principles [99]. For As, the electron Takagi's mobility and the hole Takagi's mobility were predicated to be 635 and $1700 \mathrm{~cm}^{2} \cdot \mathrm{V}^{-1} \cdot \mathrm{s}^{-1}$, respectively. For Sb, the mentioned mobilities are 630 and $1737 \mathrm{~cm}^{2} \cdot \mathrm{V}^{-1} \cdot \mathrm{s}^{-1}$, respectively. The subthreshold swing (SS) for Sb-based transistors was calculated to be $60 \mathrm{mV} \cdot \mathrm{dec}^{-1}$ with a channel length of $7 \mathrm{~nm}$, which is equal to the Boltzmann limit. These results indicate that antimonene was a competitive material for ultra-scaled transistors in the sub-10-nm scale. Ji et al. fabricated the actual transistors with top gate based on few-layered antimonene shown in Figure 2F; they observed good electrical conductivity on the order of $10^{4}$ $\mathrm{S} \cdot \mathrm{m}^{-1}$, implying the semimetal characteristic [92]. Tian et al. reported that $2 \mathrm{D}$ antimonene can be a promising anode material in sodium-ion batteries. They proposed that the few-layer antimonene can undergo anisotropic volume expansion and exhibit reversible crystalline phase evolution $\left(\mathrm{Sb} \leftrightharpoons \mathrm{NaSb} \leftrightharpoons \mathrm{Na}_{3} \mathrm{Sb}\right)$ during cycling, delivering a large capacity of $642 \mathrm{mAh} \cdot \mathrm{g}^{-1}$ at $0.1 \mathrm{C}$ [100]. Flexible PDs based on few-layered antimonene have also been fabricated, which showed a weak responsivity of $10 \mu \mathrm{A} \cdot \mathrm{W}^{-1}$ and photo-switching on/off ratio of 26.8 [101]. It was believed that the weak photoresponse is due to the indirect bandgap of $\mathrm{Sb}$. Zhang et al. realized a new class of 2D antimonene oxides with a direct bandgap and high carrier mobility. Meanwhile, antimonene nanosheetdecorated fiber laser emerged as an optical saturable absorber that can apply in the passively mode-locking and Q-switching operation at the telecommunication band [93, 96]. Moreover, antimonene oxides exhibit tunable direct bandgaps covering a wide range from 0 to $2.28 \mathrm{eV}$ (shown in Figure 2G), which is crucial for solar cell and PD applications. It is noted that antimonene oxide (18Sb-180) can be a 2D TI with a sizable global bandgap of $177 \mathrm{meV}$, adaptive for spintronic devices [102].

\subsection{Bismuth}

Bi presents a rhombohedral crystal structure, where each atom and its three equidistant nearest neighbors form a bilayer (BL) structure in the rhombohedral [103] 
direction, as shown in Figure 3A [104, 108]. As the interBL bonding is much weaker than the bonding within the $\mathrm{BL}$, epitaxial $\mathrm{Bi}(111)$ thin films were generally grown in $\mathrm{BL}$ mode. The electronic bandgap, optical gap and exciton binding energies can be tuned depending on the different crystalline orientations and the SOC effect, enabling wide application for light manipulation or modulation [109]. The bulk Bi is a semimetal with an indirect band overlap of $30-40 \mathrm{meV}$ while it undergoes a transition from semimetal to semiconductor when thinned down to be nanometer scale due to the quantum size effect $[110,111]$. It was found that an energy gap of $0.4 \mathrm{eV}$ can be formed in $\mathrm{Bi}$ (110) nanoribbons, which were grown on epitaxial graphene on $4 \mathrm{H}-\mathrm{SiC}(0001)$; the edge reconstructions can be responsible for the bandgap opening due to the edge strain energy release [110]. As a heavy element, bismuth has strong SOC on the surface, resulting in spinsplit Rashba surface states, which enables the development of spintronic devices [103, 112]. Takayama et al. fabricated the ultrathin Bi film with different thickness on $\mathrm{Si}$ (111) substrate and found the spin polarization of spin-split Rashba surface states near the Brillouin-zone boundary, which can lead to the spin-polarized electrons with tunable spin polarization [113]. Under a strong magnetic field, bismuth can also deliver unique electronic properties. For example, Li et al. have observed the sharp field-induced phase transitions of Dirac electrons into a state with striking magnetic anisotropy in Bi films [114]. Zhu et al. showed that the flow of Dirac fermions along the trigonal axis is extremely sensitive to the orientation of in-plane magnetic field, which can be utilized to tune the contribution of each valley to the total conductivity [115]. For further application of surface-state valleys in valleytronics, the electronic transport properties of the surface states in bismuth film have been studied using scanning tunneling microscopy (STM) under magnetic fields perpendicular to the surface, and specific surface spin states with a large g-factor have been identified [111].

Bismuth has also been reported as a 2D TI with nontrivial topological edge states [116, 117]. In 2D TI, a pair of topological edge states are connected with the bulk bandgap and localized at the edges with spin-up electrons
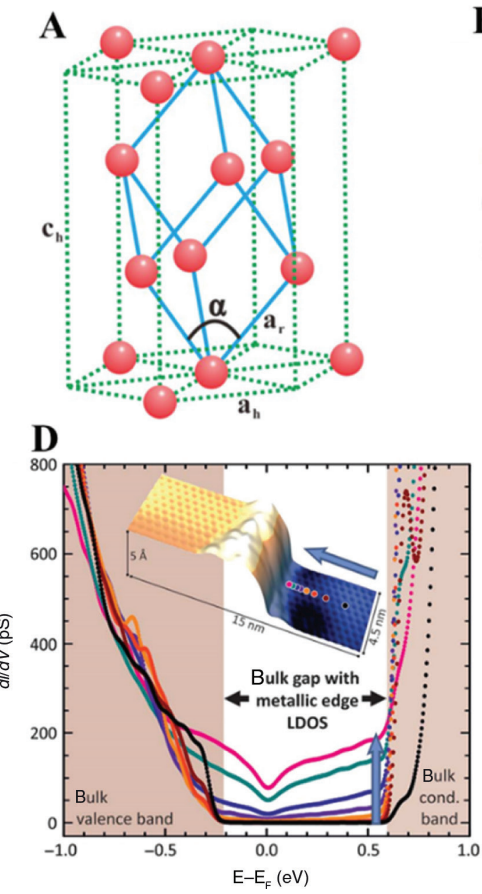

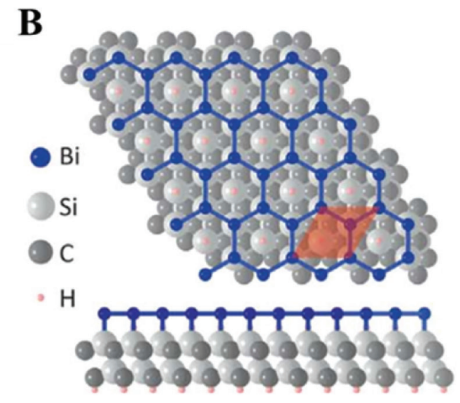

$\mathbf{E}$

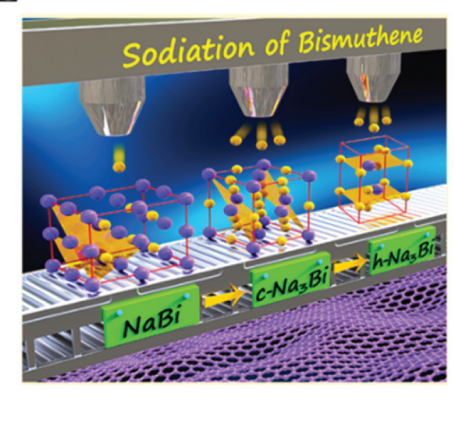

C

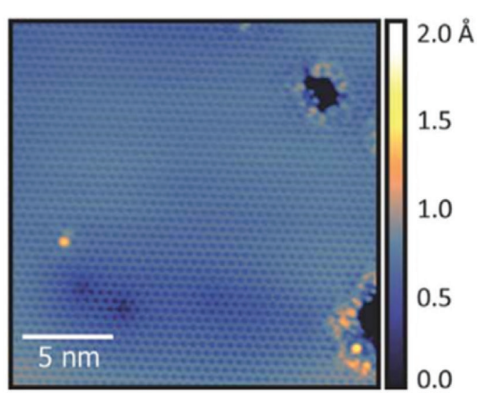

F

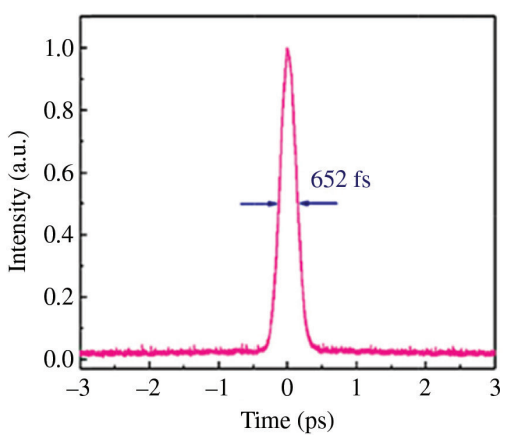

Figure 3: Structure, growth, physics and application of 2D Bi.

(A) Rhombohedra structure (blue solid line) and hexagonal structure (green dashed line) of Bi. Reproduced with permission from Ref. [104]. Copyright 2017, American Chemical Society. (B) Schematic diagram of a bismuthene layer placed on the threefold-symmetric silicon carbide (SiC) (0001) substrate [105]. (C) The honeycomb pattern of the bismuthene. (D) Differential conductivity $d I / d V$ (reflecting the LDOS) at different distances to the edge. (B-D) Reproduced with permission from Ref. [105]. Copyright 2017, The American Association for the Advancement of Science. (E) Schematic diagram of multistep phase transitions from $\mathrm{Bi} \rightarrow \mathrm{NaBi} \rightarrow \mathrm{c}-\mathrm{Na}_{3} \mathrm{Bi}$ (cubic) $\rightarrow$ h-Na $\mathrm{Bi}$ (hexagonal) during $\mathrm{Na}^{+}$intercalation and alloying processes. Reproduced with permission from Ref. [106]. Copyright 2019, American Chemical Society. (F) Autocorrelation trace of mode-locking with few-layer bismuthine. Reproduced with permission from Ref. [107]. Copyright 2017, American Chemical Society. 
and spin-down electrons propagating in the opposite direction. Thus, the 2D TI is superior to 3D TIs as the electrons at the edges of 2D TIs with opposite spins are immune to the backscattering caused by nonmagnetic defects [117]. Murakami predicted that the single-BL bismuth was 2D TI and could present the quantum spin-Hall (QSH) effect by calculating the helical edge states, which has guided the possible experimental design [116]. Afterward, freestanding Bi (111) films up to eight BLs were also predicted to be 2D TIs through a first-principles study [118]. The topological feature of 2D bismuth has provided a new platform to realize the QSH systems. The first QSH systems were realized in HgTe quantum wells by Zhang both theoretically and experimentally $[119,120]$. But the small bulk energy gap of less than $30 \mathrm{meV}$ prevents real applications. A large energy gap is desirable as the chemical potential has to reside in this gap to avoid the bulk carrier transport, and the ultralow temperature is also needed to suppress thermal excitation of charge carriers [121]. Based on the first-principles calculations, Zhou et al. demonstrated the epitaxial growth of 2D Bi TI, i.e. the QSH state, on Si (111) surface with an energy gap as large as $\sim 0.8 \mathrm{eV}$, which originates from an intriguing substrate-orbital-filtering effect [122]. Then Hsu et al. predicted a new class of 2D TIs based on planar Bi and Sb honeycombs on SiC (0001) substrate with nontrivial bandgaps of $0.56 \mathrm{eV}$. In 2017, Reis et al. achieved for the first time the monolayer bismuthine grown on a $\mathrm{SiC}(0001)$ substrate [105]. $\mathrm{A}(\sqrt{3} \times \sqrt{3}) \mathrm{R} 30^{\circ}$ superstructure of $\mathrm{Bi}$ atoms in honeycomb geometry has been generated as shown in Figure 3B. The STM shows smooth flakes with a diameter of $\sim 25 \mathrm{~nm}$ and a clear honeycomb pattern (Figure 3C). Differential tunneling conductivity $(d I / d V)$ curves, reflecting the local density of state (LDOS), have also been performed along the flake (Figure 3D), showing a large bandgap of $\sim 0.8 \mathrm{eV}$ far from the edge. The states, filling in the entire gap, emerge when closer to the edge boundary. The SiC substrate can stabilize the quasi-2D TI and play a key role for achieving the large gap, which provides a paradigm to create large-gap QSH systems.

Recently, Sun et al. prepared Bi (111) ultrathin film on the $\mathrm{NbSe}_{2}$ superconducting substrate and studied its electronic properties and edge states using angle-resolved photoemission spectroscopy (ARPES), STM and DFT calculations [104]. They observed the existence of edge states on the Bi film and the superconducting gaps from 1 $\mathrm{BL}$ to $7 \mathrm{BL}$ due to the proximity effect from the superconducting $\mathrm{NbSe}_{2}$ substrate. The coexistence of topological edge state and superconductivity in bismuth ultrathin film makes the new system a promising platform for exploring Majorana Fermions.
Besides the spintronic devices, the 2D Bi film has been reported to be a promising candidate in other application fields such as battery and photonics. Bismuth is reported to be a promising anode material for state-ofthe-art rechargeable batteries due to its high theoretical volumetric capacity and relatively low working potential [123]. In 2019, Huang et al. fabricated few-layered bismuth nanosheets by a simple probe liquid exfoliation technique and investigated the structure and phase transitions of layered bismuth during $\mathrm{Na}^{+}$intercalation and alloying processes through in situ HRTEM [106]. It was found that the few-layered bismuth nanosheets undergo the multistep phase transitions from $\mathrm{Bi} \rightarrow \mathrm{NaBi} \rightarrow \mathrm{c}$ (cubic)- $\mathrm{Na}_{3} \mathrm{Bi} \rightarrow \mathrm{h}$ (hexagonal)-Na $\mathrm{Na}_{3} \mathrm{Bi}$ and exhibits a potential cyclability for Na-ion batteries as shown in Figure 3E. At the same time, Zhou et al. also synthesized ultrathin few-layered bismuthene with a large aspect ratio and achieved a stable and high areal sodium storage capacity of $12.1 \mathrm{mAh} \cdot \mathrm{cm}^{-2}$, greatly exceeding that of most reported electrode materials [124]. Few-layered bismuthene has also been synthesized by a sonochemical exfoliation approach by Zhang's group [107]; they studied the saturable absorption property of bismuthene at the telecommunication band with an optical modulation depth of $-2.03 \%$ and a saturable intensity of $\sim 30 \mathrm{~mW} \cdot \mathrm{cm}^{-2}$. Interestingly, a $\sim 652$-femtosecond optical pulse centered at $1559.18 \mathrm{~nm}$ was achieved, as shown in Figure 3F, indicating an excellent material of bismuthene for an ultrafast saturable absorber device. Subsequently, Yang et al. developed a new strategy combining acid interaction and liquid exfoliation to successfully transform metal bulk Bi into few-layered semiconductors [125]. The 2D bismuthene was also employed as a saturable absorber to mode-lock a fiber laser and achieved a 2 $\mu \mathrm{m}$ near infrared ray (NIR)-wavelength output, revealing the strong and broad nonlinear responses of bismuthene extending into the NIR region.

The bulk Bi with a rhombohedral structure may not adopt real vdWs bonded layered structure; thus, it is challenging to exfoliate into thinner layers using the traditional top-down micromechanical exfoliation approach. Gusmão et al. have developed the aqueous shear exfoliation to obtain pnictogen (As, Sb and Bi) nanosheets [72]. In their experiments, bulk crystals of As, $\mathrm{Sb}$ and Bi were subjected to shear dispersion and exfoliation in aqueous surfactant sodium cholate for $2 \mathrm{~h}$, during which the liquid-phase shear, turbulence and collisions can lead to a gentle lateral force self-lubricating exfoliation of the pnictogen materials. Zhang and coauthors also fabricated Bi nanosheets and quantum dots by using N-methyl pyrrolidone solvent and porous anodized aluminum oxide membrane [126]. Afterward, the wet chemical method by combining acid 
interaction and ultrasonication to exfoliate grounded $\mathrm{Bi}$ into thin 2D bismuthene was also proposed, which can yield scalable 2D nanosheets [125]. On the other hand, bottom-up methods were also an alternative strategy to prepare thin Bi films. Benefiting from the low vapor pressure, ultrathin Bi film can be grown under ultrahigh vacuum [127]. Du et al. deposited the Bi (111) thin films on $\mathrm{Si}$ (111) substrates using a Knudsen cell; after evaporation, the samples were annealed at $380 \mathrm{~K}$ for about $24 \mathrm{~h}$ to produce relatively large terraces in the $\mathrm{Bi}$ (111) films [111]. Ultrathin 2D bismuth has also been fabricated on highly oriented pyrolytic graphite (HOPG) and insulating SiC (0001) to investigate their unique 2D TI properties and QSH [105, 117]. Very recently, Yang et al. demonstrated the direct growth of homogeneous and centimeter-scale layered Bi films with high-purity phase and good crystallinity by the pulsed laser deposition (PLD) technique [128]. Both Bi (111) and Bi (110) films can be readily obtained by controlling the processing temperature, showing thickness-dependent bandgaps ranging from
0.075 to $0.2 \mathrm{eV}$. Moreover, the few-layered Bi-based FETs exhibited a large mobility up to $\sim 220 \mathrm{~cm}^{2} \cdot \mathrm{V}^{-1} \cdot \mathrm{s}^{-1}$, which is superior to that in some 2D TMCs. The scalable PLD-grown Bi layers can offer a new opportunity to develop the spintronic and optoelectronic applications.

\subsection{Tellurium}

In group $\mathrm{VI}_{\mathrm{A}}$ elements, quasi-2D Te has a trigonal crystal structure, which is famous for its 1D helical/chiral chains stacking together on a 2D hexagonal lattice [129]. Because of the multivalent character of $\mathrm{Te}$, it possesses three common phases in the crystal structure, including stable- $\alpha$ (1T, space group: P2), metastable- $\beta$ (rectangular, space group: $P 2 / \mathrm{m}$, see Figure $4 \mathrm{~A}$ and $\mathrm{B})$ and $\gamma(2 \mathrm{H})$ forms $[133,134]$. There are two reasons for tellurium to be separated layer by layer via ME and epitaxial deposition. On the one hand, due to the higher cohesive energy of
A

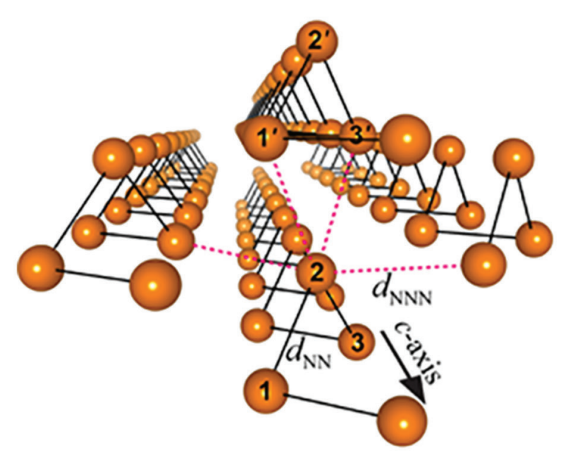

B

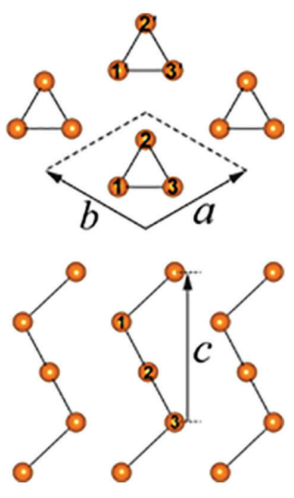

F

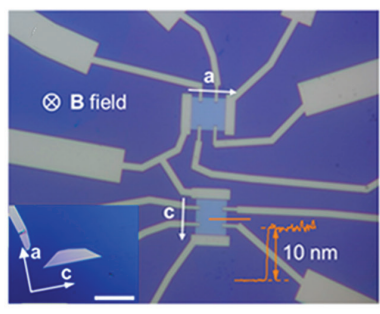

G

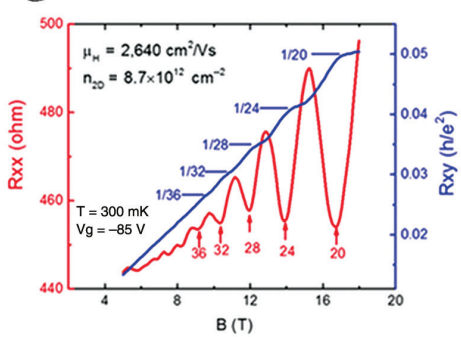

C $\mathrm{TeO}_{2}+2 \mathrm{H}_{2}=\mathrm{Te}+2 \mathrm{H}_{2} \mathrm{O}$

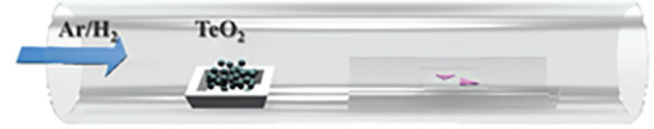

D
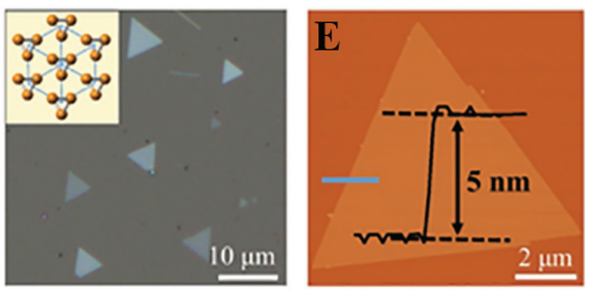

$\mathbf{H}$

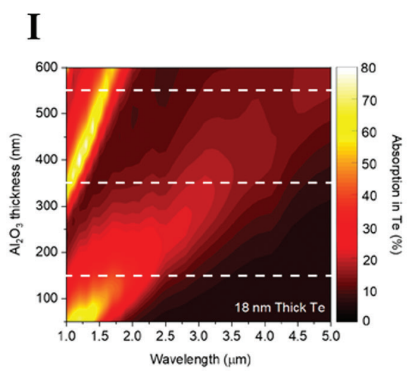

Figure 4: Structure, growth, physics and application of 2D Te.

(A) Perspective and (B) top (upper panel) and side (lower panel) views of the equilibrium structure of bulk $\beta$-Te. (A, B) Reproduced with permission from Ref. [129]. Copyright 2018, American Chemical Society. (C) Schematic diagram of the CVD method. (D) Optical image of triangular Te flakes on mica. Inset: atomic structure of tellurium along $c$-axis. (E) AFM image of a typical Te flake. (C-E) Reproduced with permission from Ref. [130]. Copyright 2019, Wiley-VCH. (F) Optical image of Hall-bar devices along two different crystal orientations fabricated from the same flake. Inset: As-grown flakes are usually in rectangular or trapezoidal shapes. The scale bar is $50 \mu \mathrm{m}$. (G) QHE and SdH oscillations measured from a 10-nm-thick flake. (F, G) Reproduced with permission from Ref. [131]. Copyright 2018, American Chemical Society. $(\mathrm{H})$ Schematic showing the optical cavity structure used to fabricate SWIR photodetectors from quasi-2D Te nanoflakes. An evaporated Au film acts as the gate electrode and back-reflector, while an $\mathrm{Al}_{2} \mathrm{O}_{3}$ dielectric layer acts as $\sim \lambda / 4$ spacer as well as the gate oxide. (I) Calculated absorption in the Te layer, as a function of wavelength and $\mathrm{Al}_{2} \mathrm{O}_{3}$ thickness for a fixed semiconductor thickness of $18 \mathrm{~nm}$. ( $\left.\mathrm{H}, \mathrm{I}\right)$ Reproduced with permission from Ref. [132]. Copyright 2018, American Chemical Society. 
$0.06 \mathrm{eV}$ than that of $1 \mathrm{D}$ chains, Te crystals with different stacking type tend to grow into 2D nanostructures [135]. On the other hand, although the covalent bonds form in 1D chiral chains, the interaction between neighboring tellurene layers is of vdWs type [133]. Additionally, bulk, nanobelt and nanowire forms of $\mathrm{Te}$ are demonstrated at an early stage. 2D layered tellurium or tellurene materials have been comprehensively explored by a number of researchers in recent years.

In 2014, Wang et al. was the first to prepare 2D tellurium nanoplates on flexible mica substrates via a vdWs epitaxy method. However, the thickness of Te is beyond $30 \mathrm{~nm}$ and the lateral dimensions are in the range of 6-10 $\mu \mathrm{m}$ [136]. After that, Te nanostructures based on other substrate such as sapphire, graphene/4H-SiC and HOPG have also be studied in recent years [137-143]. In general, there are many kinds of synthetic methods, including physical vapor deposition (PVD) [135], MBE [138], LPE [144-146], hydrothermal reaction [131, 132, 147, 148], CVD [130] and thermal evaporation deposition [149, 150]. Without consideration of the crystal scale, both Lin et al. and Wu et al. demonstrated free-standing exfoliated Te nanosheets at the size of several hundred nanometers in polarized solvent by ultrasonic bath and centrifugation $[144,146]$. To date, they found greater air stability and biocompatibility of Te nanosheets after the functionalization of glutathione (GSH), which can be used in the photodynamic therapy agents. In order to be used in (opto) electronic devices, in 2018, Wu et al. comprehensively and systematically fabricated 2D tellurium nanosheets through the reduction of sodium tellurite $\left(\mathrm{Na}_{2} \mathrm{TeO}_{3}\right)$ by hydrazine hydrate $\left(\mathrm{N}_{2} \mathrm{H}_{4}\right)$ in an alkaline solution at temperatures from 160 to $200^{\circ} \mathrm{C}$, by adding a crystal-face-blocking ligand polyvinylpyrrolidone [147]. A similar fabrication can be seen in the as-prepared Te nanowires by Hunyadi et al. [140]. 2D quadrangle Te nanosheets from monolayer to multilayer with a lateral size of $10-50 \mu \mathrm{m}$ have been successfully achieved at different conditions [147]. Furthermore, in 2019, Zhang et al. prepared triangular 2D Te flakes on mica substrate via a creative CVD, in the presence of $\mathrm{TeO}_{2}$ with the mixture gas of $\mathrm{Ar} / \mathrm{H}_{2}$. The thinnest thickness can reach approximately $5 \mathrm{~nm}$ (see Figure 4C-E) [130]. Freshly, Zhao et al. developed a thermal deposition method at cryogenic temperature to receive large-area polycrystalline Te nanosheets, showing unprecedented FET performance [150]. Above all, 2D Te is a promising material for wafer-scale production either through solution reaction or through CVD and thermal deposition (at cryogenic temperature).

Unlike the metastable of 2D arsenene, 2D tellurium nanosheets possess excellent air stability almost in the whole thickness range. Several studies of the unencapsulated Te devices in a moderate thickness range $(>3 \mathrm{~nm}$ ) have shown no obvious degradation for a long time such as 1 or 2 months $[131,139,150]$. One of the intrinsic reasons is that an energy barrier exists for the correlated oxidation pathways and it can prevent the few-layered Te from being oxidized under ambient conditions [151]. In addition, $\beta$-Te is more stable for monolayer while $\alpha$-Te cannot maintain and transfer to the $\beta$ phase without a barrier. For band structure, based on the Perdew-Burke-Ernzerhofer plus SOC calculation, $\alpha$-Te and $\beta$-Te deliver semiconducting behaviors with an indirect bandgap of 0.46 and $1.03 \mathrm{eV}$, respectively [133]. In contrast, $\gamma$-Te is metal. Moreover, for comparison, the experimental bandgap of Te can be tuned from $0.32 / 0.33 \mathrm{eV}$ to $0.92 \mathrm{eV}$ (direct bandgap) from bulk to monolayer due to the quantum confinement effect [139, 152, 153]. Most importantly, wave function hybridization occurs for covalent-like quasi-bonding (CLQB) as well as the interchain interaction of few-layered $\mathrm{Te}$, leading to ultrahigh theoretical hole mobility $\left(10^{4}-10^{6} \mathrm{~cm}^{2} \cdot \mathrm{V}^{-1} \cdot \mathrm{s}^{-1}\right)$ and strong visible-infrared light absorption along the CLQB direction [154]. Nevertheless, experimental mobility is affected by gate efficiency, impurity scattering, charge traps, contact and so on. Until now, the reported maximum hole mobility is $\sim 700 \mathrm{~cm}^{2} \cdot \mathrm{V}^{-1} \cdot \mathrm{s}^{-1}$ at room temperature at the $2 \mathrm{D}$ Te thickness of $\sim 16 \mathrm{~nm}$ by solution exfoliation [147]. The hole mobility can be significantly increased by gate voltage and cryogenic temperature. As is known, QSH is satisfied in confined 2D electron gas and occurred in highmobility 2DLMs with a large external magnetic field at low temperature. Reasonably, the magnetic transport behavior of 2D Te with high mobility is reported and probably brings a novel phenomenon along different directions $[131,155]$. The TI behavior found in 2D $\alpha$-Te over the 3-nm region was determined by STM [148], already theoretical explained in strain engineering for Te nanowires and nanosheets [143, 154, 156]. For optical behavior, 2D Te with a thickness from $9.1 \mathrm{~nm}$ to $20.5 \mathrm{~nm}$ showed four red-shifted Raman peaks of $94 \mathrm{~cm}^{-1}\left(\mathrm{E}_{1} \mathrm{TO}\right), 105 \mathrm{~cm}^{-1}\left(\mathrm{E}_{1}\right.$ LO), $125 \mathrm{~cm}^{-1}\left(\mathrm{~A}_{1}\right)$ and $143 \mathrm{~cm}^{-1}\left(\mathrm{E}_{2}\right)$, attributed to the augmentation of deformation potential in lattice orientation and the interlayer long-range Coulombic interactions $[130,147]$. Meanwhile, $E_{1}$ TO and $E_{1}$ LO Raman modes are shifted and disappeared for few-layered and multilayered 2D Te, respectively $[147,157]$. In contrast, Te nanowires prepared by MBE always showed the above typical Raman peaks [158]. Therefore, Raman spectrum is a highly reliable technology to confirm the thickness range and chiralchain structure of Te. In view of the 1D helical chain along the c-axis and vdWs-like between chains in the triangular structure, 2D Te nanosheets exhibit unique anisotropy in 
bandgap, carrier mobility, phonon vibrations and optical absorption in the infrared region [132, 147, 154]. The angleresolved Raman peak intensities of different modes were observed in 13.5-nm Te nanosheets. However, the anisotropic hole-mobility ratio of $15 \mathrm{~nm} 2 \mathrm{D}$ Te was only $\sim 1.5$ due to the enhanced surface scattering [147]. It is necessary to figure out the anisotropy behavior for multilayer Te nanosheets in Raman and electronics. Interestingly, a 18.8-nm-thick 2D Te-based PD showed isotropic and anisotropic responsivity at wavelengths of 1.5 and $3 \mu \mathrm{m}$, respectively. It is indicated that the high anisotropy of the indirect bandgap was achieved for 2D Te nanosheet, while the direct bandgap was independent of the polarization of the infrared light [132]. Distinctly, Te also possesses an obvious photothermal effect in a wide broadband spectrum because of its narrow bandgap, which is already used in photothermal cancer therapy [159].

Given the various physics of 2D tellurium and tellurene, such as flexibility, stability, high mobility, narrow bandgap and high optical absorption, their applications in FETs [130, 137, 147, 150], photodynamic therapy [144], optical modulator [146], polarized infrared imaging [132], quantum information [131, 155], PDs [130, 132, 136] and solar absorber [159] have been explored in recent years. In 2018, Wu et al. showed that thickness-dependent 2D Te FETs exhibited the recorded highest hole mobility of $700 \mathrm{~cm}^{2} \cdot \mathrm{V}^{-1} \cdot \mathrm{s}^{-1}$ with a moderate on/off ratio of $\sim 10^{3}$ at ambient conditions. Furthermore, they also found that a short-channel device at 7-8-nm-thick Te showed the best performance, with an on/off ratio of $10^{4}$ and maximum drain current of $>600 \mathrm{~mA} \cdot \mathrm{mm}^{-1}$ [147]. To promote largescale processing and the application of $2 \mathrm{D} \mathrm{Te}$, Zhao et al. prepared high-performance wafer-scale polycrystalline Te FETs, logic gates and computational circuit in 2019 by low-temperature deposition method [150]. In their results, the statistical performance for 60 devices at a Te thickness of $\sim 8 \mathrm{~nm}$ offered values of $32 \pm 7 \mathrm{~cm}^{2} \cdot \mathrm{V}^{-1} \cdot \mathrm{s}^{-1}$ for mobility,
$9491 \pm 4765$ for on/off current ratio and $114 \pm 9 \mathrm{mV} \cdot \mathrm{dec}^{-1}$ for SS at low drain bias. It is indicated that the as-prepared polycrystalline Te film showed negligible defects and high crystal quality. Moreover, the electrical properties of the flexible device showed no significant change after 500 cycles of bending at a radius of $6 \mathrm{~mm}$, suggesting that this Te film can be used in flexible and transparent electronics and displays [160]. Under a tilted magnetic field, Qiu et al. was the first to report direct observation of quantum Hall effect in 2D Te with fourfold degeneracy because of the interplay of Zeeman splitting and strong Rashba-like SOC in Te. The spintronics showed ultrahigh Hall mobility of nearly $3000 \mathrm{~cm}^{2} \cdot \mathrm{V}^{-1} \cdot \mathrm{s}^{-1}$ at $0.3 \mathrm{~K}$ with a gate voltage of -80 $\mathrm{V}$ (see Figure $4 \mathrm{~F}$ and $\mathrm{G}$ ). In addition, the short-wave infrared (SWIR) PDs showed a peak responsivity from $13 \mathrm{~A} \cdot \mathrm{W}^{-1}$ $(1.4 \mu \mathrm{m})$ to $8 \mathrm{~A} \cdot \mathrm{W}^{-1}(2.4 \mu \mathrm{m})$ with a cutoff wavelength of 3.4 $\mu \mathrm{m}$ via an optical $\mathrm{Al}_{2} \mathrm{O}_{3}$ cavity structure (see Figure $4 \mathrm{H}$ and I) [132]. This result is almost consistent with the indirect bandgap of $0.35 \mathrm{eV}$ in bulk and the direct bandgap of 1.04 $\mathrm{eV}$ in monolayer for Te. Above all, with the development of the facile growth and theoretical predication, 2D Te-based nanodevices have been demonstrated to exhibit various and novel physics and photonics applications.

\subsection{Selenium}

Inspired by the development of 2D Te possessing strong in-plane anisotropic properties and high carrier mobility [161, 162], Se from group VI in a 2D form has emerged very recently. Se has trigonal atomic structures where the Se atoms are covalently connected in a spiral chain along the c-axis with two adjacent atoms rotated by $120^{\circ}$, as shown in Figure 5A. Because of the anisotropic chain-like crystal structure, Se tends to form 1D structures and has considerable properties such as photoconductivity, piezoelectricity and nonlinear optics [164-166]. In 2017, Qin et al. achieved
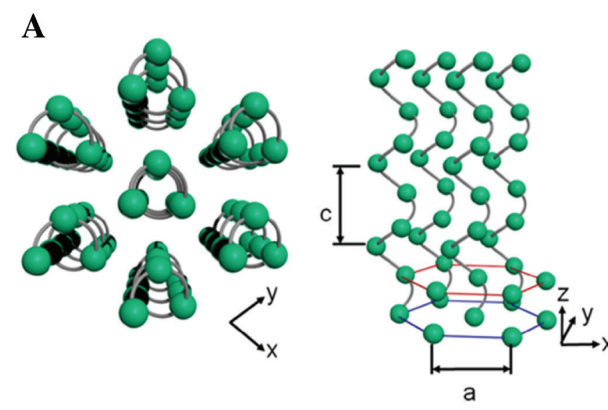

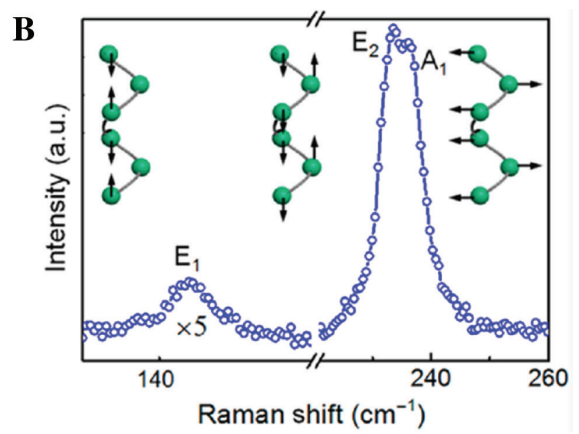

Figure 5: Structure and physics of 2D Se.

(A) Atomic structures of selenium. (B) Raman spectral of selenium nanosheets. Reproduced with permission from Ref. [163]. Copyright 2017, American Chemical Society. 
large-size, high-quality 2D selenium nanosheets with a thickness of $\sim 5 \mathrm{~nm}$ trough a PVD method [163]. From the Raman spectrum (Figure 5B), two typical Raman peaks located at $\sim 233$ and $237 \mathrm{~cm}^{-1}$, which can be assigned to the $\mathrm{E}_{2}$ and $\mathrm{A}_{1}$ vibration modes, respectively, have been identified. Similar to 2D tellurium, the selenium nanosheets also exhibit strong in-plane anisotropic properties from the angle-resolved Raman spectra. The FETs based on the selenium nanosheets were also fabricated, displaying p-type behavior with a high current on/off ratio exceeding $10^{6}$ and low hole mobility of $0.26 \mathrm{~cm}^{2} \cdot \mathrm{V}^{-1} \cdot \mathrm{s}^{-1}$. Photoresponsivity can reach up to $263 \mathrm{~A} \cdot \mathrm{W}^{-1}$ with a fast response speed of $0.1 \mathrm{~s}$. Then, Zhang's group developed a facile LPE method to synthesize 2D Se nanosheets with 40-120 nm lateral dimension and 3-6 $\mathrm{nm}$ thickness [167, 168]. The bandgap varied from 1.98 to $2.31 \mathrm{eV}$ depending on the thickness. They also applied the 2D Se nanosheets into an optical modulation device, demonstrating the excellent ultrashort pulse generation of an optical communication band. Although rarely reported, 2D Se holds promise in the future of electronic and optical applications, with benefits from its sizable bandgap, high stability and facile fabrication.

\section{Ternary materials}

In contrast to the widely reported 2D elemental and binary materials, 2D ternary layered compounds have emerged as a new 2D platform for numerous applications due to their more adjustable physical and chemical properties because of the additional element species.

\section{$3.1 \mathrm{Bi}_{2} \mathrm{O}_{2} \mathrm{Se}$}

In recent years, owing to their excellent high mobility and air stability, layered bismuth oxyselenide $\left(\mathrm{Bi}_{2} \mathrm{O}_{2} \mathrm{Se}\right)$ has emerged as a new 2D semiconductor and is expected to be applied in future optoelectronic. $\mathrm{Bi}_{2} \mathrm{O}_{2} \mathrm{Se}$ is a typical bismuth-based oxychalcogenide material; the crystal structure is presented in Figure 6A. As can be seen, $\mathrm{Bi}_{2} \mathrm{O}_{2} \mathrm{Se}$ belongs to the $14 / \mathrm{mmm}$ space group, possessing a layered tetragonal structure with $\mathrm{a}=3.88 \AA \mathrm{A}, \mathrm{c}=12.16$ $\AA$ and $Z=2$ [170]. The crystal structure consists of alternatively stacked $\left[\mathrm{Bi}_{2} \mathrm{O}_{2}\right]$ layers and Se square net layers along the c-axis, and the thickness of a layer is $0.61 \mathrm{~nm}$ [175]. In 2017, on an atomically flat fluorophlogopite mica substrate, Peng's group firstly achieved sub-millimeter single-crystalline $\mathrm{Bi}_{2} \mathrm{O}_{2} \mathrm{Se}$ nanoplates through a CVD method (Figure 6B) $[170,172]$. As presented in Figure 6C, the as-synthesized samples show a square shape with a large size over $0.2 \times 0.2 \mathrm{~mm}^{2}$. The atomic force microscopy measurement in Figure 6D confirms that the sample's surface is ultraflat and the thickness is $\sim 6.7 \mathrm{~nm}$. Then, by using TEM measurement and selected area electron diffraction patterns, they further determined that the prepared samples belonged to the quadrilateral $I 4 / \mathrm{mmm}$ space group (see Figure 6E). Next, through ARPES and first-principles calculations, they investigated the band structure of the $\mathrm{Bi}_{2} \mathrm{O}_{2} \mathrm{Se}$ crystals. As presented in Figure 6F, the $\mathrm{Bi}_{2} \mathrm{O}_{2} \mathrm{Se}$ is measured to possess an indirect bandgap of $\sim 0.8 \mathrm{eV}$. This value is consistent with the calculated one of $\sim 0.85 \mathrm{eV}$ (Figure $6 \mathrm{G}$ ). This bandgap is suitable for photodetection over a wide spectral range. In addition, a low in-plane electron effective mass of $m^{\star}=0.14 \pm 0.02 m_{0}$ is extracted. This $m^{\star}$ value is superior to that of traditional $\mathrm{Si}\left(0.26 \mathrm{~m}_{0}\right)$, BP $\left(0.15 \mathrm{~m}_{0}\right)$ and $\mathrm{MoS}_{2}$ $\left(0.4-0.6 \mathrm{~m}_{0}\right)$, suggesting the possibility of achieving ultrahigh electron mobility. Then, through magnetic transport measurements, a strong spin-orbit interaction was found, and a high Hall mobility up to $28,900 \mathrm{~cm}^{2} \cdot \mathrm{V}^{-1} \cdot \mathrm{s}^{-1}$ was evaluated at $1.9 \mathrm{~K}$ (see Figure 6H) [176]. Benefiting from such high-mobility electrons, Shubnikov-de Haas $(\mathrm{SdH})$ quantum oscillations were successfully observed (Figure 6I). Furthermore, FETs based on this $\mathrm{Bi}_{2} \mathrm{O}_{2} \mathrm{Se}$ also exhibit superior properties (large current on/off ratios $>10^{6}$ and almost ideal SS values of $\left.\sim 65 \mathrm{mV} \cdot \mathrm{dec}^{-1}\right)$. In 2018 , Chen et al. verified the electronic structures of $\mathrm{Bi}_{2} \mathrm{O}_{2} \mathrm{Se}$ and reported a higher mobility reaching $\sim 2.8 \times 10^{5} \mathrm{~cm}^{2} \cdot \mathrm{V}^{-1} \cdot \mathrm{s}^{-1}$ at $2 \mathrm{~K}$ by adopting the ME method [177]. In recent years, the photodetection potential of $\mathrm{Bi}_{2} \mathrm{O}_{2} \mathrm{Se}$ phototransistors covering the ultraviolet (UV)-Vis-NIR spectral range has also been extensively investigated. Liu's group reported utrasensitive $\mathrm{Bi}_{2} \mathrm{O}_{2}$ Se phototransistors with a responsivity up to $3.5 \times 10^{4} \mathrm{~A} \cdot \mathrm{W}^{-1}$ at $532 \mathrm{~nm}$, while the dark current was just several pA [169]. Tian et al. focused on nonlinear absorption of polycrystalline $\mathrm{Bi}_{2} \mathrm{O}_{2} \mathrm{Se}$, demonstrating an ultrabroadband optical switch covering the NIR and mid-infrared ray ranges [178]. Yin et al. reported an infrared $\mathrm{PD}$ based on $2 \mathrm{D} \mathrm{Bi}_{2} \mathrm{O}_{2} \mathrm{Se}$, which demonstrated a high sensitivity of $65 \mathrm{~A} \cdot \mathrm{W}^{-1}$ at $1200 \mathrm{~nm}$ and ultrafast photoresponse of $\sim 1 \mathrm{ps}$ at room temperature (Figure $6 \mathrm{~J}$ ) [171]. Through a modified CVD method, Tong et al. synthesized large-area $(\approx 180 \mu \mathrm{m}) \mathrm{Bi}_{2} \mathrm{O}_{2}$ Se nanosheets and constructed PDs [54]. The photodetection wavelength of the device covers the UV-Vis-NIR range, and the R reaches $108,696 \mathrm{~A} \cdot \mathrm{W}^{-1}$ (at $360 \mathrm{~nm}$ ), 50,055 A $\cdot \mathrm{W}^{-1}$ (at $405 \mathrm{~nm}$ ), $25,505 \mathrm{~A} \cdot \mathrm{W}^{-1}$ (at $532 \mathrm{~nm}$ ), $843.5 \mathrm{~A} \cdot \mathrm{W}^{-1}$ (at $808 \mathrm{~nm}$ ), 118 $\mathrm{A} \cdot \mathrm{W}^{-1}($ at $1310 \mathrm{~nm})$ and $22.12 \mathrm{~A} \cdot \mathrm{W}^{-1}($ at $1550 \mathrm{~nm})$. Moreover, the response time was $32 \mu$ s (Figure $6 \mathrm{~K}$ ). Wu et al. obtained centimeter-scale well-ordered 2D $\mathrm{Bi}_{2} \mathrm{O}_{2} \mathrm{Se}$ 

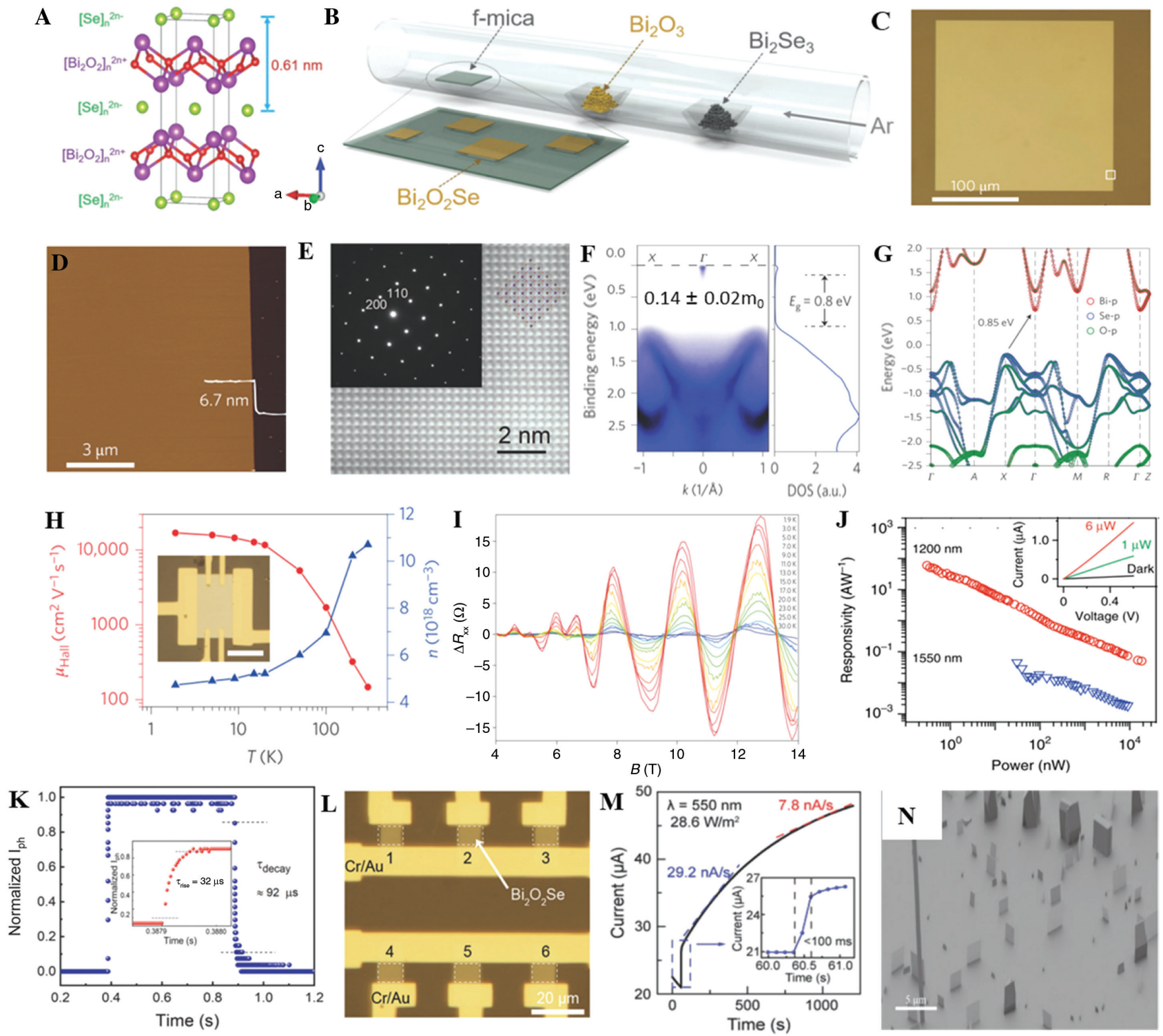

Figure 6: Structure, growth, physics and application of $2 \mathrm{D} \mathrm{Bi}_{2} \mathrm{O}_{2} \mathrm{Se}$.

(A) Crystal structure of $\mathrm{Bi}_{2} \mathrm{O}_{2}$ Se. (B) Schematic of the CVD setup for the synthesis of $\mathrm{Bi}_{2} \mathrm{O}_{2} \mathrm{Se}$. (A, B) Reproduced with permission from Ref. [169]. Copyright 2018, Wiley-VCH. (C) Optical microscopy image of a $\mathrm{Bi}_{2} \mathrm{O}_{2}$ Se nanoplate with a domain size larger than $200 \mu \mathrm{m}$. (D) Corresponding AFM image recorded at the rectangular area highlighted in C. (C, D) Reproduced with permission from Ref. [170]. Copyright 2018, Sringer Nature. (E) High-resolution TEM image of the $\mathrm{Bi}_{2} \mathrm{O}_{2}$ Se nanoplate. The SAED pattern (inset) shows that the nanoplate is single-crystalline. Reproduced with permission from Ref. [54]. Copyright 2019, Wiley-VCH. (F) Band structure of bulk $\mathrm{Bi}_{2} \mathrm{O}_{2} \mathrm{Se}$ observed by ARPES measurements. An indirect bandgap of $\sim 0.8 \mathrm{eV}$ is clearly observable. (G) Calculated band structure of $\mathrm{Bi}_{2} \mathrm{O}_{2} \mathrm{Se}_{2}$ with a bandgap of $\sim 0.85 \mathrm{eV}$. (H) Hall mobility $\left(\mu_{\text {Hall }}\right)$ and carrier density as a function of temperature in a $\mathrm{Bi}_{2} \mathrm{O}_{2}$ Se nanoplate. Inset: optical microscopy image of a Hall-bar device fabricated on the $\mathrm{Bi}_{2} \mathrm{O}_{2}$ Se nanoplate. (I) SdH oscillatory part of the longitudinal magnetoresistance $\Delta \mathrm{R}_{x x}$ as a function of applied perpendicular magnetic field measured in the temperature range from 1.9 to $30 \mathrm{~K}$. (F-I) Reproduced with permission [170].

(J) Photoresponsivity at wavelengths of $1200 \mathrm{~nm}$ and $1500 \mathrm{~nm}$. Reproduced with permission from Ref. [171]. Copyright 2018, Springer Nature. (K) Time-resolved photoresponse of $\mathrm{Bi}_{2} \mathrm{O}_{2} \mathrm{Se}$. Inset: $\tau_{\text {rise }}$ is approximately $32 \mu \mathrm{s}$. Reproduced with permission from Ref. [54]. Copyright 2019, Wiley-VCH. (L) Optical microscopy image of $2 \mathrm{D} \mathrm{Bi}_{2} \mathrm{O}_{2}$ Se integrated optoelectronic devices on mica, and each channel was labeled with a specific number from 1 to 6. Reproduced with permission from Ref. [172]. Copyright 2018, Springer Nature. (M) Time evolution of the device current after one light excitation. Reproduced with permission from Ref. [173]. Copyright 2019, Wiley-VCH. (N) Magnified view of vertical grown $\mathrm{Bi}_{2} \mathrm{O}_{2}$ Se nanoplates with a seed layer. Reproduced with permission from Ref. [174]. Copyright 2019, Wiley-VCH.

arrays with tailorable configurations [172]. Then, based on these $2 \mathrm{D} \mathrm{Bi}_{2} \mathrm{O}_{2} \mathrm{Se}$ arrays, integrated PDs were constructed (Figure 6L), which shows excellent air stability and high photoresponsivity of $\approx 2000 \mathrm{~A} \cdot \mathrm{W}^{-1}$ at $532 \mathrm{~nm}$. Furthermore, Yang et al. found that the photoconductive effect and the bolometric effect can coexist in $\mathrm{Bi}_{2} \mathrm{O}_{2} \mathrm{Se}$ 
PDs (Figure 6M) [173]. With the increase in incident light power, the thermal effect in $\mathrm{Bi}_{2} \mathrm{O}_{2}$ Se devices gradually dominates. Sequentially, to eliminate the influence of the substrate on the $\mathrm{Bi}_{2} \mathrm{O}_{2} \mathrm{Se}$, Wu et al. adopted $\mathrm{Bi}_{2} \mathrm{O}_{3}$ as a seed layer and realized vertical growth of $2 \mathrm{D} \mathrm{Bi}_{2} \mathrm{O}_{2} \mathrm{Se}$ on mica substrates (Figure 6N) [174]. Then, for the first time, the intrinsic thermal properties of $2 \mathrm{D} \mathrm{Bi}_{2} \mathrm{O}_{2}$ Se were revealed (about $1 \mathrm{~W} \cdot \mathrm{m}^{-1} \cdot \mathrm{k}^{-1}$ ). Li's group firstly used $2 \mathrm{D} \mathrm{Bi}_{2} \mathrm{O}_{2}$ Se for memristors [179]. In their report, truly concomitant shortterm and long-term plasticities were firstly demonstrated, opening up the prospects for ultrathin, high-speed and low-power neuromorphic devices.

\section{2 $\operatorname{BiOX}(X=C l, B r, I)$}

The ternary $\mathrm{Bi}_{2} \mathrm{O}_{2}$ Se materials exhibit ultrahigh mobility and novel physical properties and can be applied in infrared PDs as discussed above [176, 177]. Subsequently, new 2D ternary semiconducting materials have attracted increasing attention for various applications [180, 181]. As a representative layered ternary material, the bismuth oxyhalides (BiOX, $\mathrm{X}=\mathrm{Cl}, \mathrm{Br}$, and I) have favorable band structures for energy harvesting and optoelectronics. BiOX belongs to a group of V-VI-VII ternary compounds, possessing tetragonal matlockite structure with the $\mathrm{P4} /$ $\mathrm{nmm}$ space group [182]. As shown in Figure 7A, the monolayer of $\mathrm{BiOX}$ comprises one slab of $\left[\mathrm{Bi}_{2} \mathrm{O}_{2}\right]^{2+}$ interleaved by double slabs of halogen ions $[\mathrm{X}]^{-}$forming the $[\mathrm{X}-\mathrm{Bi}-\mathrm{O}$ $\mathrm{Bi}-\mathrm{X}]$ stacking. Zhao et al. predicted that the BiOI has a bandgap of $\approx 2.2 \mathrm{eV}$ and is appropriate for the overall photocatalytic water-splitting reaction when the thickness is less than $3 \mathrm{~nm}$ because of the higher conduction band edge than the reduction potential of $\mathrm{H}^{+} / \mathrm{H}^{2}$ [184]. Hoye et al. synthesized BiOI thin films by the CVD method and demonstrated their experimental bandgap of $1.9 \mathrm{eV}$ and promising photovoltaic applications [185].

Recently, the band structures of a series of bismuth/ antimony oxyhalides and chalcohalides have been investigated in detail, which suggest their great potential as solar absorbers, transparent conductors and radiation detectors [186]. In 2019, Zeng et al. achieved large-area BiOI from monolayer to few layers using the space-confined CVD method [183]. Figure 7B shows an optical microscopy image of the typical BiOI layers with square shape and lateral size exceeding $130 \mu \mathrm{m}$. By introducing the oxygen vacancies under the ultraviolet light irradiation, the PDs based on few-layered BiOI can exhibit much improved performances with $\mathrm{I}_{\text {on }} / \mathrm{I}_{\text {off }}$ ratio and detectivity up to $1 \times 10^{5}$ and $8.2 \times 10^{11}$ Jones at $473 \mathrm{~nm}$, respectively. Similarly, 2D BiOCl has also been developed, demonstrating a relatively larger bandgap close to $3.2 \mathrm{eV}$ [187]. Kang et al. reported a very fast and reliable approach to the synthesis of 2D ternary-layered BiOCl crystals by the space-confined microwave-assisted heating process where $\mathrm{BiCl}_{3} \bullet \mathrm{H}_{2} \mathrm{O}$ was heated by a molecular interaction with the electromagnetic field and then decomposed to square shape $\mathrm{BiOCl}$ flakes with edge size up to $10 \mu \mathrm{m}$ and thickness of $248 \mathrm{~nm}$ on the $\mathrm{SiO}_{2} / \mathrm{Si}$ substrate [188]. The asobtained BiOCl platelets exhibit high performance of photodetection to 266-nm laser with responsivity up to $\sim 8 \mathrm{~A} \cdot \mathrm{W}^{-1}$

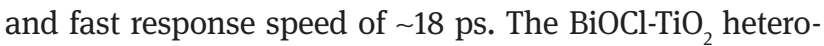
junctions were also explored as ultraviolet PDs performing ultrahigh on/off ratio (up to $2.2 \times 10^{5}$ ), responsivity of 41.94 $\mathrm{A} \cdot \mathrm{W}^{-1}$ and detectivity $\left(\mathrm{D}^{*}\right)$ of $1.41 \times 10^{14}$ Jones, as well as a high linear dynamic range of $103.59 \mathrm{~dB}$ [189]. Overall, the favorable band structures, facile CVD growth and high stability suggest that 2D BiOX can be a promising material for future optoelectronic applications.

\subsection{Ternary 2D ferromagnetic materials}

Due to the low energy consumption, fast operation and high storage density, spintronic devices hold a
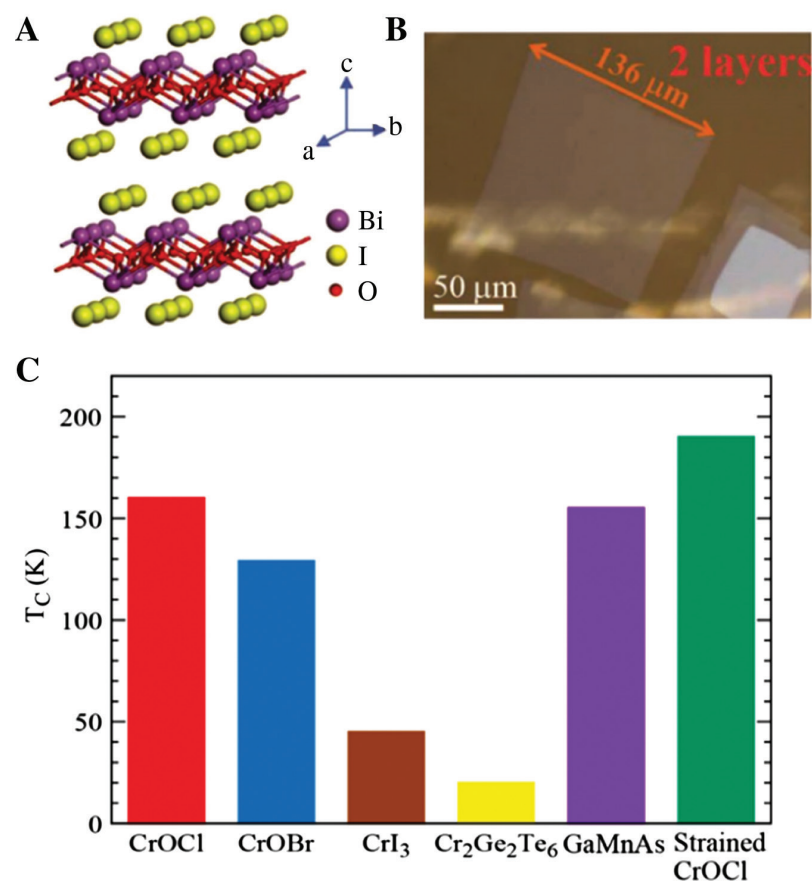

Figure 7: Structure, growth and physics of 2D BiOX and CrOX. (A) Layered crystal structure of BiOl with stacked [I-Bi-O-Bi-I] slabs. (B) Optical microscopy images of large-area atomically thin $\mathrm{BiOI}$ nanosheets. Reproduced with permission from Ref. [183]. Copyright 2019, Wiley-VCH. (C) Curie temperature in comparison with $2 \mathrm{D} \mathrm{Crl}_{3}$, $\mathrm{Cr}_{2} \mathrm{Ge}_{2} \mathrm{Te}_{6}$, GaMnAs and single-layer $\mathrm{CrOCl}$ under $5 \%$ biaxial tensile strain. Reproduced with permission from Ref. [53]. Copyright 2018, American Chemical Society. 
huge development room in future quantum information technology. The prerequisite to realize the spintronics is the intrinsically ferromagnetic $2 \mathrm{D}$ semiconductor. 2D binary $\mathrm{CrI}_{3}$ and $\mathrm{CrBr}_{3}$ have been successfully demonstrated as strong ferromagnetic materials by several groups. Jiang et al. reported that the monolayer $\mathrm{CrI}_{3}$ is a ferromagnet (FM) while $\mathrm{BL} \mathrm{CrI}_{3}$ is an antiferromagnet, the magnetic properties can be tuned by electrostatic doping using $\mathrm{CrI}_{3}$-graphene vertical heterostructures [190]. Huang et al. reported similar work that demonstrated the electrostatic gate control of magnetism in $\mathrm{CrI}_{3} \mathrm{BLs}$, allowing a gate-voltage-driven phase transition from a layered antiferromagnet phase to a FM phase [191]. A giant tunneling magnetoresistance of $19,000 \%$ was also achieved using the few-layer $\mathrm{CrI}_{3}$ as the spin-filter tunnel barrier sandwiched between graphene contacts [192]. The Curie temperature $\left(\mathrm{T}_{\mathrm{c}}\right)$ of $\mathrm{CrI}_{3}$ is reported to be $45 \mathrm{~K}$ [193], which is still lower than the liquid $\mathrm{N}_{2}$ temperature limiting, its further applications. Recently, Bonilla et al. found a strong ferromagnetic ordering in monolayer $\mathrm{VSe}_{2}$, and the large magnetic moment can persist to above room temperature, making $\mathrm{VSe}_{2}$ an attractive material for spintronic applications at room temperature [194]. However, a common issue for the above-discussed binary 2D ferromagnetic materials is the poor stability in atmosphere ambient. Thus, research interests have focused on the ferromagnetic ternary materials in the same time trying to address this issue and further improving the $\mathrm{T}_{\mathrm{c}}$ value. Gong et al. discovered the intrinsic long-range ferromagnetic order in pristine $\mathrm{Cr}_{2} \mathrm{Ge}_{2} \mathrm{Te}_{6}$ atomic layers with a very low $\mathrm{T}_{c}$ of $20 \mathrm{~K}$ [195]. The $\mathrm{T}_{c}$ of $\mathrm{Cr}_{2} \mathrm{Ge}_{2} \mathrm{Te}_{6}$ was further increased to $65 \mathrm{~K}$ via gate electrical doping [196]. Subsequently, Deng et al. developed a new device fabrication technique to successfully achieve the monolayer $\mathrm{Fe}_{3} \mathrm{GeTe}_{2}$. They found that the monolayer $\mathrm{Fe}_{3} \mathrm{GeTe}_{2}$ possesses an itinerant ferromagnetism with an out-of-plane magneto-crystalline anisotropy and the $\mathrm{T}_{\mathrm{c}}$ can be raised to room temperature by an ionic gate, offering a new opportunity for potential voltage-controlled magneto-electronics [197].

Very recently, the monolayer chromium oxyhalide (CrOX, $\mathrm{X}=\mathrm{Cl}$ or $\mathrm{Br}$ ) has emerged as a new class of 2D spintronic semiconductors with intrinsic ferromagnetism, large spin polarization and high Curie temperature up to 160 and $129 \mathrm{~K}$, respectively, making 2D CrOX a new promising platform for future spintronics [53]. More importantly, both the $\mathrm{CrOCl}$ and $\mathrm{CrOBr}$ monolayers have great dynamic and thermal stability according to their phonon dispersions and molecular dynamic trajectories. Figure 7C shows the $\mathrm{T}_{\mathrm{c}}$ of CrOX in comparison with $2 \mathrm{D} \mathrm{CrI}_{3}(45 \mathrm{~K}$ ), $\mathrm{Cr}_{2} \mathrm{Ge}_{2} \mathrm{Te}_{6}(20 \mathrm{~K})$ and GaMnAs (155 K). It is found that the $\mathrm{T}_{\mathrm{c}}$ of the CrOX monolayers can exceed the liquid nitrogen temperature (77 K), which can be further improved to be $204 \mathrm{~K}$ by applying appropriate strains. Moreover, layered ytterbium oxychloride (YbOCl), also as a member of the MOX family, has been synthesized successfully by Xia et al. [198]. The prepared hexagonal and triangular shaped YbOCl nanosheets with a thickness of $\sim 10 \mathrm{~nm}$ showed different Raman anisotropic behaviors. Further studies can be focused on its ferromagnetic properties as theoretical prediction.

\section{Outlook and conclusion}

As discussed above, the novel monoelemental and ternary 2D materials have shown a promising future in electronics, photonics, electrodes, batteries and biomedicine.

Among them, photonics (such as PDs) and energy (such as solar absorbers, energy storage and thermoelectrics) applications for monoelemental materials need to be further studied. On the other hand, they are all at the early development stage, and some issues should be addressed in order to further improve their performance and promote their practical applications (see Figure 8).

\subsection{Stability}

In general, it is often inevitable for most $2 \mathrm{D}$ materials to interact with the surroundings (gas, liquid), so their experimental performance and application range can be limited to some extent. For example, a serious

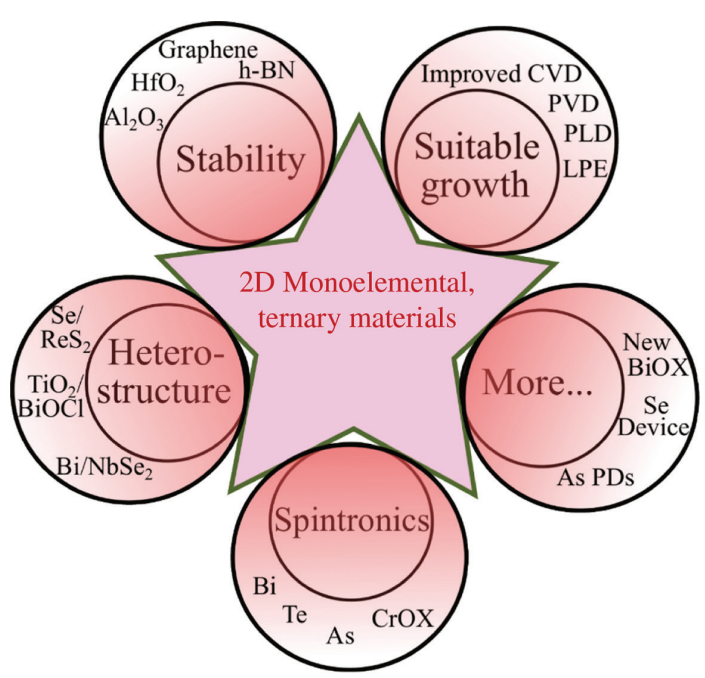

Figure 8: Summary of the novel 2D monoelemental, ternary materials in this paper. 
disadvantage of BP is its poor air stability, and all operations, including synthesis, device fabrication and testing, have to be performed in an inert atmosphere, which has led to the restriction of processing technologies, daily storage and wide applications. A similar issue also exists for some new monoelemental and ternary materials such as black As, $\mathrm{Cr}_{2} \mathrm{Ge}_{2} \mathrm{Te}_{6}$, etc. Some strategies such as alloy engineering (B-AsP) [20, 21] and allotrope synthesis (Violet phosphorus) [22] have been proposed to resolve the problem. Another possible choice is the formation of vdWs heterostructures encapsulated with h-BN, graphene or surface passivated with a high- $k$ insulator layer such as $\mathrm{HfO}_{2}, \mathrm{Al}_{2} \mathrm{O}_{3}$, etc. In addition, solvent polymer layers such as PMMA and PEG are also considered for use in protecting unstable 2D materials. Fortunately, monoelemental Te, $\mathrm{Bi}, \mathrm{Sb}$ and Se as well as ternary $\mathrm{Bi}_{2} \mathrm{O}_{2} \mathrm{Se}, \mathrm{BiOX}$ and $\mathrm{CrOX}$ nanosheets possess very robust environmental stability, which is more adaptive for large-scale and longterm applications in the future.

\subsection{Suitable growth method}

Large-scale growth or processing is instrumental in order to realize wide commercial applications in modern electronics and optoelectronics, which is dependent on the perfect matching of crystal lattice between 2D materials and the supporting substrate. The growth of graphene and TMCs $\left(\mathrm{MoS}_{2}, \mathrm{WS}_{2}\right.$, etc.) has been very mature, delivering wafer-scale production and complementary MOScompatible device arrays. Simultaneously, improved CVD, PVD, PLD and magnetron sputtering methods to prepare large-area 2D materials have been developed well. So far, the common methods used to synthesize 2D monoelemental and ternary materials are LPE or micromechanical exfoliation, yielding a very small size ranging from hundreds of nanometers to tens of micrometers and bringing a number of foreign atoms or by-products, whereas for biomedical application, a large quantity of biocompatible 2D materials with small size are suitable and efficient, such as novel titanium nanosheets prepared by the LPE method [199]. Thus, the discussed 2D monoelemental materials in this paper can be extended to be used in drug therapy and carriers such as GSH functionalized Te nanosheets [144]. Due to the anisotropic rhombohedral or trigonal crystal structure of buckled elemental materials, it is challenging to achieve uniform and large-area 2D nanosheets through the solid precursors. Epitaxial growth in a high-vacuum condition on different substrates (such as mica, sapphire, $\mathrm{Si}, \mathrm{Ag}, \mathrm{Cu}$ ) has been developed to get high-quality nanosheets with relatively large-size materials but still on the order of micrometers. Thus, the wafer-scale growth of novel 2D monoelemental and ternary materials remains a great opportunity for future research. Meanwhile, for the 2D materials in this paper, crystalline orientation, controllable production and growth mechanism on different substrates or solvents should also be studied further. Efficient growth strategies are urgently required for the fabrication of device arrays and integrated chips in the future.

\subsection{Performance improvement}

Currently, numerous theoretical works have predicted high device performances such as the intrinsic high carrier mobility of 2D As and $\mathrm{Sb}$, and ternary CrOX was also predicted to possess intrinsic ferromagnetism, large spin polarization and high Curie temperature. However, experimental works have rarely been carried out or the obtained devices exhibit much lower performance than the theoretical value. For example, the predicted hole mobility of b-As can exceed $10^{3} \mathrm{~cm}^{2} \cdot \mathrm{V}^{-1} \cdot \mathrm{s}^{-1}$; however, the reported maximum experimental value is only $\sim 59 \mathrm{~cm}^{2} \cdot \mathrm{V}^{-1} \cdot \mathrm{s}^{-1}$ as a result of the poor material quality and pristine device structure. Thus, it is believed that the strategies utilized to improve graphene- or TMC-based devices, such as band or dielectric engineering, can be applied to the new monoelemental and ternary materials. Moreover, the newly discovered topological edge states and long-term ferromagnetism in bismuthine, arsenene, tellurium and CrOX hold promise in spintronic devices such as spin FETs, spin light-emitting diodes, and QSH systems. The ingenious combination with other 2D materials to form heterostructures via epitaxial growth or dry-transfer method can also offer a diversity of platforms to study new physics and devices. For instance, the heterostructures based on topological bismuthene and superconducting materials such as $\mathrm{NbSe}_{2}$ can be utilized to exploit the theoretically predicted Majorana fermions. Moreover, similar to $\mathrm{WS}_{2} /$ $\mathrm{CsPbBr}$, the hybridization of some stable monoelemental materials with 2D materials (such as TMCs) is also a potential strategy to enhance optoelectronic performance [200-202]. For example, Ye et al. developed the epitaxial growth of Se nanoplates on the monolayer $\mathrm{ReS}_{2}$, where the obtained heterostructures exhibit ultrahigh optoelectronic performance [202].

In summary, we discussed the newly emerging 2D monoelemental (As, Sb, Bi, Te and Se) and ternary $\left(\mathrm{Bi}_{2} \mathrm{O}_{2} \mathrm{Se}, \mathrm{BiOX}, \mathrm{CrOX}\right.$, etc.) materials in term of their structure, growth, physics and applications. Compared to the widely studied graphene, BP, TMCs, etc., these $2 \mathrm{D}$ 
materials have the advantages of favorable band structure, good air stability, unique physics and high optical and electrical properties, offering new possibilities in future nanodevices. Of course, further efforts should be taken on the existing issues to realize their real commercial applications.

Acknowledgments: This work was financially supported by the National Natural Science Foundation of China (11904108, Funder Id: http://dx.doi. org/10.13039/501100001809, 61805045 and 61805044), "The Pearl River Talent Recruitment Program" and the "Hundred Talents Program" of Guangdong University of Technology (GDUT).

Conflicts of interest: The authors declare no conflicts of interest.

\section{References}

[1] Novoselov KS, Geim AK, Morozov SV, et al. Electric field effect in atomically thin carbon films. Science 2004;306:666-9.

[2] Geim AK, Novoselov KS. The rise of graphene. Nanosci Technol A Collection Rev Nat J 2010;11-9.

[3] Kauling AP, Seefeldt AT, Pisoni DP, et al. The worldwide graphene flake production. Adv Mater 2018;30:1803784.

[4] Lin L, Peng H, Liu Z. Synthesis challenges for graphene industry. Nat Mater 2019;18:520-9.

[5] Cao Y, Fatemi V, Fang S, et al. Unconventional superconductivity in magic-angle graphene superlattices. Nature 2018;556:43-50.

[6] Yang Y, Yang X, Liang L, et al. Large-area graphene-nanomesh/ carbon-nanotube hybrid membranes for ionic and molecular nanofiltration. Science 2019;364:1057-62.

[7] You R, Liu YQ, Hao YL, et al. Laser fabrication of graphenebased flexible electronics. Adv Mater 2019;1901981.

[8] Polat EO, Mercier G, Nikitskiy I, et al. Flexible graphene photodetectors for wearable fitness monitoring. Sci Adv 2019;5: eaaw7846.

[9] Leong WS, Wang H, Yeo J, et al. Paraffin-enabled graphene transfer. Nat Commun 2019;10:867.

[10] Sun L, Lin L, Wang Z, et al. A force-engineered lint roller for superclean graphene. Adv Mater 2019;1902978.

[11] Yuhara J, He B, Matsunami N, et al. Graphene's latest cousin: plumbene epitaxial growth on a "nano watercube". Adv Mater 2019;1901017.

[12] Yang S, Zhang K, Ricciardulli AG, et al. A delamination strategy for thinly layered defect-free high-mobility black phosphorus flakes. Angew Chem Int Ed 2018;57:4677-81.

[13] Liu S, Huo N, Gan S, et al. Thickness-dependent Raman spectra, transport properties and infrared photoresponse of few-layer black phosphorus. J Mater Chem C 2015;3:10974-80.

[14] Kim S, Cui J, Dravid VP, et al. Orientation-dependent intercalation channels for lithium and sodium in black phosphorus. Adv Mater 2019;1904623.
[15] Zhang Y, Zhang F, Xu Y, et al. Self-healable black phosphorus photodetectors. Adv Funct Mater 2019;1906610.

[16] Gao A, Lai J, Wang Y, et al. Observation of ballistic avalanche phenomena in nanoscale vertical InSe/BP heterostructures. Nat Nanotechnol 2019;14:217.

[17] Lin Z, Wang J, Guo X, et al. Interstitial copper-doped edge contact for $\mathrm{n}$-type carrier transport in black phosphorus. InfoMat 2019;1:242-50.

[18] Zhou Y, Zhang M, Guo Z, et al. Recent advances in black phosphorus-based photonics, electronics, sensors and energy devices. Mater Horiz 2017;4:997-1019.

[19] Hu T, Hong J. Anisotropic effective mass, optical property, and enhanced band gap in BN/phosphorene/BN heterostructures. ACS Appl Mater Interfaces 2015;7:23489-95.

[20] Long M, Gao A, Wang P, et al. Room temperature high-detectivity mid-infrared photodetectors based on black arsenic phosphorus. Sci Adv 2017;3:e1700589.

[21] Guo S, Zhang Y, Ge Y, et al. 2D V-V binary materials: status and challenges. Adv Mater 2019;31:1902352.

[22] Zhang L, Huang H, Zhang B, et al. Structure and properties of violet phosphorus and its phosphorene exfoliation. Angew Chem Int Ed 2019;132:1090-6.

[23] Ge Y, Chen S, Xu Y, et al. Few-layer selenium-doped black phosphorus: synthesis, nonlinear optical properties and ultrafast photonics applications. J Mater Chem C 2017;5:6129-35.

[24] Huo N, Yang Y, Li J. Optoelectronics based on 2D TMDs and heterostructures. J Semicond 2017;38:031002.

[25] Huo N, Konstantatos G. Recent progress and future prospects of 2D-based photodetectors. Adv Mater 2018;30:1801164.

[26] Yan F, Wei Z, Wei X, et al. Toward high-performance photodetectors based on 2D materials: strategy on methods. Small Methods 2018;2:1700349.

[27] Zhang X, Lai Z, Ma Q, et al. Novel structured transition metal dichalcogenide nanosheets. Chem Soc Rev 2018;47:3301-38.

[28] Huo N, Konstantatos G. Ultrasensitive all-2D MoS 2 phototransistors enabled by an out-of-plane $\mathrm{MoS}_{2}$ PN homojunction. Nat Commun 2017;8:572.

[29] Geng D, Yang HY. Recent advances in growth of novel 2D materials: beyond graphene and transition metal dichalcogenides. Adv Mater 2018;30:1800865.

[30] Long $M$, Wang P, Fang $\mathrm{H}$, et al. Progress, challenges, and opportunities for 2D material based photodetectors. Adv Funct Mater 2019;29:1803807.

[31] Cheng J, Wang C, Zou X, et al. Recent advances in optoelectronic devices based on 2D materials and their heterostructures. Adv Opt Mater 2019;7:1800441.

[32] Pi L, Li L, Liu K, et al. Recent Progress on 2D noble-transitionmetal dichalcogenides. Adv Funct Mater 2019;29:1904932.

[33] Xie Z, Zhang F, Liang Z, et al. Revealing of the ultrafast third-order nonlinear optical response and enabled photonic application in two-dimensional tin sulfide. Photonics Res 2019;7:494-502.

[34] Wu L, Xie Z, Lu L, et al. Few-layer tin sulfide: a promising blackphosphorus-analogue 2D material with exceptionally large nonlinear optical response, high stability, and applications in all-optical switching and wavelength conversion. Adv Opt Mater 2018;6:1700985.

[35] Huang W, Xie Z, Fan T, et al. Black-phosphorus-analogue tin monosulfide: an emerging optoelectronic two-dimensional material for high-performance photodetection with improved 
stability under ambient/harsh conditions. J Mater Chem C 2018;6:9582-93.

[36] Xie Z, Wang D, Fan T, et al. Black phosphorus analogue tin sulfide nanosheets: synthesis and application as near-infrared photothermal agents and drug delivery platforms for cancer therapy. J Mater Chem B 2018;6:4747-55.

[37] Gao W, Zheng ZQ, Li Y, et al. High performance tin diselenide photodetectors dependent on thickness: vertical graphene sandwiched device and interfacial mechanism. Nanoscale 2019;11:13309.

[38] Huang B, Yoon M, Sumpter BG, et al. Alloy engineering of defect properties in semiconductors: Suppression of deep levels in transition-metal dichalcogenides. Phys Rev Lett 2015;115:126806.

[39] Rhodes D, Chae SH, Ribeiro-Palau R, et al. Disorder in van der Waals heterostructures of 2D materials. Nat Mater 2019;18:541.

[40] Liu Y, Gao Z, Tan Y, et al. Enhancement of out-of-plane charge transport in a vertically stacked two-dimensional heterostructure using point defects. ACS Nano 2018;12:10529-36.

[41] Tongay S, Suh J, Ataca C, et al. Defects activated photoluminescence in two-dimensional semiconductors: interplay between bound, charged, and free excitons. Sci Rep-UK 2013;3:2657.

[42] Avsar A, Ciarrocchi A, Pizzochero M, et al. Defect induced, layer-modulated magnetism in ultrathin metallic $\mathrm{PtSe}_{2}$. Nat Nanotechnol 2019;14:674-8.

[43] Li B, Xing T, Zhong M, et al. A two-dimensional Fe-doped $\mathrm{SnS}_{2}$ magnetic semiconductor. Nat Commun 2017;8:1958.

[44] Liu J, Zhong M, Liu X, et al. Two-dimensional plumbum-doped tin diselenide monolayer transistor with high on/off ratio. Nanotechnology 2018;29:474002.

[45] Liu J, Liu X, Chen Z, et al. Tunable Schottky barrier width and enormously enhanced photoresponsivity in $\mathrm{Sb}$ doped $\mathrm{SnS}_{2}$ monolayer. Nano Res 2019;12:463-8.

[46] Zhong M, Shen C, Huang L, et al. Electronic structure and exciton shifts in Sb-doped $\mathrm{MoS}_{2}$ monolayer. NPJ 2D Mater Appl 2019;3:1.

[47] Kong X, Liu Q, Zhang C, et al. Elemental two-dimensional nanosheets beyond graphene. Chem Soc Rev 2017;46:2127-57.

[48] Glavin NR, Rao R, Varshney V, et al. Emerging applications of elemental 2D materials. Adv Mater 2019;1904302.

[49] Song Y, You K, Chen Y, et al. Lead monoxide: a promising two-dimensional layered material for applications of nonlinear photonics at infrared band. Nanoscale 2019;11:12595.

[50] Cui F, Feng Q, Hong J, et al. Synthesis of large-size $1 \mathrm{~T}^{\prime}$ $\operatorname{ReS}_{2 x} \mathrm{Se}_{2(1-x)}$ alloy monolayer with tunable bandgap and carrier type. Adv Mater 2017;29:1705015.

[51] Tongay S, Narang DS, Kang J, et al. Two-dimensional semiconductor alloys: monolayer $\mathrm{Mo}_{1-\mathrm{x}} \mathrm{W}_{\mathrm{x}} \mathrm{Se}_{2}$. Appl Phys Lett 2014;104:012101.

[52] Mounet N, Gibertini M, Schwaller P, et al. Two-dimensional materials from high-throughput computational exfoliation of experimentally known compounds. Nat Nanotechnol 2018;13:246.

[53] Miao N, Xu B, Zhu L, et al. 2D intrinsic ferromagnets from Van der Waals antiferromagnets. J Am Chem Soc 2018;140:2417-20.

[54] Tong T, Chen Y, Qin S, et al. Sensitive and ultrabroadband phototransistor based on two-dimensional $\mathrm{Bi}_{2} \mathrm{O}_{2}$ Se nanosheets. Adv Funct Mater 2019;29:1905806.

[55] Wang YW, Liu S, Zeng BW, et al. Ultraviolet saturable absorption and ultrafast carrier dynamics in ultrasmall black phosphorus quantum dots. Nanoscale 2017;9:4683-90.
[56] He J, Tao L, Zhang H, et al. Emerging 2D materials beyond graphene for ultrashort pulse generation in fiber lasers. Nanoscale 2019;11:2577-93.

[57] Sang DK, Wang H, Guo Z, et al. Recent developments in stability and passivation techniques of phosphorene toward next-generation device applications. Adv Funct Mater 2019;29:1903419.

[58] Tao W, Kong N, Ji X, et al. Emerging two-dimensional monoelemental materials (Xenes) for biomedical applications. Chem Soc Rev 2019;48:2891.

[59] Luo K, Chen SY, Duan CG. Indirect-direct band gap transition of two-dimensional arsenic layered semiconductors-cousins of black phosphorus. Sci China Phys Mech 2015;58:87301.

[60] Clark AH. Arsenolalnprite confirmed from the Copiapó area, northern Chile. Mineral Mag 1970;37:732-3.

[61] Wu F, Li Q, Wang P, et al. High efficiency and fast van der Waals hetero-photodiodes with a unilateral depletion region. Nat Commun 2019;10:1-8.

[62] Zhong M, Xia Q, Pan L, et al. Thickness-dependent carrier transport characteristics of a new 2D elemental semiconductor: black arsenic. Adv Funct Mater 2018;28:1802581.

[63] Chen B, Xue L, Han Y, et al. Structural, mechanical, and electronic properties of nanotubes based on buckled arsenene: $a$ first-principles study. Mater Today Commun 2019;22:100791.

[64] Beladi-Mousavi SM, Pourrahimi AM, Sofer Z, et al. Atomically thin 2D-arsenene by liquid-phased exfoliation: toward selective vapor sensing. Adv Funct Mater 2019;29:1807004.

[65] Hu Y, Qi ZH, Lu J, et al. Van der Waals epitaxial growth and interfacial passivation of two-dimensional single-crystalline fewlayer gray arsenic nanoflakes. Chem Mater 2019;31:4524-35.

[66] Jamdagni P, Thakur A, Kumar A, et al. Two dimensional allotropes of arsenene with a wide range of high and anisotropic carrier mobility. Phys Chem Chem Phys 2018;20:29939-50.

[67] Chen Y, Chen C, Kealhofer R, et al. Black arsenic: a layered semiconductor with extreme in-plane anisotropy. Adv Mater 2018;30:1800754.

[68] Matsubara S, Miyawaki R, Shimizu M, et al. Pararsenolamprite, a new polymorph of native As, from the Mukuno mine, Oita Prefecture Japan. Mineralogic Mag 2001;65:807-12.

[69] Wcykoff GMG. Crystal structures. New York: Wiley, 1963.

[70] Yoshiasa A, Tokuda M, Misawa M, et al. Natural arsenic with a unique order structure: potential for new quantum materials. Sci Rep-UK 2019;9:6275.

[71] Sturala J, Ambrosi A, Sofer Z, et al. Covalent functionalization of exfoliated arsenic with chlorocarbene. Angew Chem 2018;130:15053-6.

[72] Gusmão R, Sofer Z, Bouša D, et al. Pnictogen (As, Sb, Bi) nanosheets for electrochemical applications are produced by shear exfoliation using kitchen blenders. Angew Chem 2017;129:14609-14.

[73] Vishnoi P, Mazumder M, Pati SK, et al. Arsenene nanosheets and nanodots. New J Chem 2018;42:14091-5.

[74] Sturala J, Sofer Z, Pumera M. Chemistry of layered pnictogens: phosphorus, arsenic, antimony, and bismuth. Angew Chem 2019;131:7631-7.

[75] Wang Y, Huang P, Ye M, et al. Many-body effect, carrier mobility, and device performance of hexagonal arsenene and antimonene. Chem Mater 2017;29:2191-201.

[76] Kou L, Ma Y, Tan X, et al. Structural and electronic properties of layered arsenic and antimony arsenide. J Phys Chem C 2015;119:6918-22. 
[77] Wang X, Li Y, Huang L, et al. Short-wave near-infrared linear dichroism of two-dimensional germanium selenide. J Am Chem Soc 2017;139:14976-82.

[78] Zhou Z, Long M, Pan L, et al. Perpendicular optical reversal of the linear dichroism and polarized photodetection in 2D GeAs. ACS Nano 2018;12:12416-23.

[79] Pumera M, Sofer Z. 2D monoelemental arsenene, antimonene, and bismuthene: beyond black phosphorus. Adv Mater 2017;29:1605299.

[80] Zhou Z, Cui Y, Tan PH, et al. Optical and electrical properties of two-dimensional anisotropic materials. J Semicond 2019;40:061001.

[81] Liu B, Köpf M, Abbas AN, et al. Black arsenic-phosphorus: layered anisotropic infrared semiconductors with highly tunable compositions and properties. Adv Mater 2015;27:4423-9.

[82] Zhang Z, Xie J, Yang D, et al. Manifestation of unexpected semiconducting properties in few-layer orthorhombic arsenene. Appl Phys Express 2015;8:055201.

[83] Arsat R, Breedon M, Shafiei M, et al. Graphene-like nanosheets for surface acoustic wave gas sensor applications. Chem Phys Lett 2009;467:344-7.

[84] Liu C, Liu CS, Yan X. Arsenene as a promising candidate for $\mathrm{NO}$ and $\mathrm{NO}_{2}$ sensor: a first-principles study. Phys Lett A 2017;381:1092-6.

[85] Li L, Han W, Pi L, et al. Emerging in-plane anisotropic twodimensional materials. InfoMat 2019;1:54-73.

[86] Ares P, Palacios JJ, Abellán G, et al. Recent progress on antimonene: a new bidimensional material. Adv Mater 2018;30:1703771.

[87] Zhang S, Yan Z, Li Y, et al. Atomically thin arsenene and antimonene: semimetal-semiconductor and indirect-direct band-gap transitions. Angew Chem Inter Edit 2015;54:3112-5.

[88] Wang G, Pandey R, Karna SP. Atomically thin group V elemental films: theoretical investigations of antimonene allotropes. ACS Appl Mater Interfaces 2015;7:11490-6.

[89] Peng L, Ye S, Song J, et al. Solution-phase synthesis of fewlayer hexagonal antimonene nanosheets via anisotropic growth. Angew Chem Int Ed 2019;58:9891-6.

[90] Shao Y, Liu ZL, Cheng C, et al. Epitaxial growth of flat antimonene monolayer: a new honeycomb analogue of graphene. Nano Lett 2018;18:2133-9.

[91] Zhu SY, Shao Y, Wang E, et al. Evidence of topological edge states in buckled antimonene monolayers. Nano Lett 2019;19:6323-9.

[92] Ji J, Song X, Liu J, et al. Two-dimensional antimonene single crystals grown by van der Waals epitaxy. Nat Commun 2016;7:13352.

[93] Song Y, Shi X, Wu C, et al. Recent progress of study on optical solitons in fiber lasers. Appl Phys Rev 2019;6:021313.

[94] Ares P, Aguilar-Galindo F, Rodríguez-San-Miguel D, et al. Mechanical isolation of highly stable antimonene under ambient conditions. Adv Mater 2016;28:6332-3.

[95] Gibaja C, Rodriguez-San-Miguel D, Ares P, et al. Back cover: few-layer antimonene by liquid-phase exfoliation. Angew Chem Int Ed 2016;55:14470.

[96] Song Y, Liang Z, Jiang X, et al. Few-layer antimonene decorated microfiber: ultra-short pulse generation and all-optical thresholding with enhanced long term stability. 2D Mater 2017;4:045010.
[97] Xue T, Liang W, Li Y, et al. Ultrasensitive detection of miRNA with an antimonene-based surface plasmon resonance sensor. Nat Commun 2019;10:1-9.

[98] Fortin-Deschênes $M$, Waller 0 , Mentes TO, et al. Synthesis of antimonene on germanium. Nano Lett 2017;17:4970-5.

[99] Pizzi G, Gibertini M, Dib E, et al. Performance of arsenene and antimonene double-gate MOSFETs from first principles. Nat Commun 2016;7:12585.

[100] Tian W, Zhang S, Huo C, et al. Few-layer antimonene: anisotropic expansion and reversible crystalline-phase evolution enable large-capacity and long-life $\mathrm{Na}$-ion batteries. ACS Nano 2018;12:1887-93.

[101] Xiao Q, Hu CX, Wu HR, et al. Antimonene-based flexible photodetector. Nanoscale Horiz 2020;5:124-30.

[102] Zhang S, Zhou W, Ma Y, et al. Antimonene oxides: emerging tunable direct bandgap semiconductor and novel topological insulator. Nano Lett 2017;17:3434-40.

[103] Hirahara T, Miyamoto K, Matsuda I, et al. Direct observation of spin splitting in bismuth surface states. Phys Rev B 2007;76:153305.

[104] Sun HH, Wang MX, Zhu F, et al. Coexistence of topological edge state and superconductivity in bismuth ultrathin film. Nano Lett 2017;17:3035-9.

[105] Reis F, Li G, Dudy L, et al. Bismuthene on a SiC substrate: a candidate for a high-temperature quantum spin Hall material. Science 2017;357:287-90.

[106] Huang Y, Zhu C, Zhang S, et al. Ultrathin bismuth nanosheets for stable Na-Ion batteries: clarification of structure and phase transition by in situ observation. Nano Lett 2019;19:1118-23.

[107] Lu L, Liang Z, Wu L, et al. Few-layer bismuthene: sonochemical exfoliation, nonlinear optics and applications for ultrafast photonics with enhanced stability. Laser Photonics Rev 2018;12:1700221.

[108] Hofmann P. The surfaces of bismuth: Structural and electronic properties. Prog Surf Sci 2006;81:191-45.

[109] Kecik D, Özçelik VO, Durgun E, et al. Structure dependent optoelectronic properties of monolayer antimonene, bismuthene and their binary compound. Phys Chem Chem Phys 2019;21:7907-17.

[110] Sun JT, Huang $\mathrm{H}$, Wong SL, et al. Energy-gap opening in a Bi (110) nanoribbon induced by edge reconstruction. Phys Rev Lett 2012;109:246804.

[111] Du H, Sun X, Liu X, et al. Surface Landau levels and spin states in bismuth (111) ultrathin films. Nat Commun 2016;7:10814.

[112] Koroteev YM, Bihlmayer G, Gayone JE, et al. Strong spin-orbit splitting on Bi surfaces. Phys Rev Lett 2004;93:046403.

[113] Takayama A, Sato T, Souma S, et al. Tunable spin polarization in bismuth ultrathin film on Si (111). Nano Lett 2012;12:1776-9.

[114] Li L, Checkelsky JG, Hor YS, et al. Phase transitions of Dirac electrons in bismuth. Science 2008;321:547-50.

[115] Zhu Z, Collaudin A, Fauqué B, et al. Field-induced polarization of Dirac valleys in bismuth. Nat Phys 2012;8:89.

[116] Murakami S. Quantum spin Hall effect and enhanced magnetic response by spin-orbit coupling. Phys Rev Lett 2006;97:236805.

[117] Lu Y, Xu W, Zeng M, et al. Topological properties determined by atomic buckling in self-assembled ultrathin $\mathrm{Bi}(110)$. Nano Lett 2014;15:80-7. 
[118] Liu Z, Liu CX, Wu YS, et al. Stable nontrivial Z topology in ultrathin Bi (111) films: a first-principles study. Phys Rev Lett 2011;107:136805.

[119] Bernevig BA, Hughes TL, Zhang SC. Quantum spin Hall effect and topological phase transition in HgTe quantum wells. Science 2006;314:1757-61.

[120] König M, Wiedmann S, Brüne C, et al. Quantum spin Hall insulator state in $\mathrm{HgTe}$ quantum wells. Science 2007;318:766-70.

[121] Väyrynen JI, Goldstein M, Glazman LI. Helical edge resistance introduced by charge puddles. Phys Rev Lett 2013;110:216402.

[122] Zhou M, Ming W, Liu Z, et al. Epitaxial growth of large-gap quantum spin Hall insulator on semiconductor surface. P Natl Acad Sci USA 2014;111:14378-81.

[123] Lei K, Wang C, Liu L, et al. A porous network of bismuth used as the anode material for high-energy-density potassium-ion batteries. Angew Chem Inter Edit 2018;57:4687-91.

[124] Zhou J, Chen J, Chen M, et al. Few-Layer Bismuthene with Anisotropic Expansion for High-Areal-Capacity Sodium-Ion Batteries. Adv Mater 2019; 31:1807874.

[125] Yang QQ, Liu RT, Huang C, et al. 2D bismuthene fabricated via acid-intercalated exfoliation showing strong nonlinear near-infrared responses for mode-locking lasers. Nanoscale 2018;10:21106-15.

[126] Xing C, Huang W, Xie Z, et al. Ultrasmall bismuth quantum dots: facile liquid-phase exfoliation, characterization, and application in high-performance UV-Vis photodetector. ACS Photonics 2017;5:621-9.

[127] Scott SA, Kral MV, Brown SA. A crystallographic orientation transition and early stage growth characteristics of thin $\mathrm{Bi}$ films on HOPG. Surf Sci 2005;587:175-84.

[128] Yang Z, Wu Z, Lyu Y, et al. Centimeter-scale growth of two-dimensional layered high-mobility bismuth films by pulsed laser deposition. InfoMat 2019;1:98-107.

[129] Yi S, Zhu Z, Cai X, et al. The nature of bonding in bulk tellurium composed of one-dimensional helical chains. Inorg Chem 2018;57:5083-8.

[130] Zhang X, Jiang J, Suleiman AA, et al. Hydrogen-assisted growth of ultrathin Te flakes with giant gate-dependent photoresponse. Adv Funct Mater 2019;1906585.

[131] Qiu G, Wang Y, Nie Y, et al. Quantum transport and band structure evolution under high magnetic field in few-layer tellurene. Nano Lett 2018;18:5760-7.

[132] Amani M, Tan C, Zhang G, et al. Solution-synthesized high-mobility tellurium nanoflakes for short-wave infrared photodetectors. ACS Nano 2018;12:7253-63.

[133] Zhu Z, Cai X, Yi S, et al. Multivalency-driven formation of Te-based monolayer materials: a combined first-principles and experimental study. Phys Rev Lett 2017;119:106101.

[134] Wu B, Liu X, Yin J, et al. Bulk $\beta$-Te to few layered $\beta$-tellurenes: indirect to direct band-gap transitions showing semiconducting property. Mater Res Express 2017;4:095902.

[135] Apte A, Bianco E, Krishnamoorthy A, et al. Polytypism in ultrathin tellurium. 2D Mater 2018;6:015013.

[136] Wang Q, Safdar M, Xu K, et al. Van der Waals epitaxy and photoresponse of hexagonal tellurium nanoplates on flexible mica sheets. ACS Nano 2014;8:7497-05.

[137] Sapkota KR, Lu P, Medlin DL, et al. High temperature synthesis and characterization of ultrathin tellurium nanostructures. APL Mater 2019;7:081103.
[138] Chen J, Dai Y, Ma Y, et al. Ultrathin $\beta$-tellurium layers grown on highly oriented pyrolytic graphite by molecular-beam epitaxy. Nanoscale 2017;9:15945-8.

[139] Huang X, Guan J, Lin Z, et al. Epitaxial growth and band structure of Te film on graphene. Nano Lett 2017;17:4619-23.

[140] Hunyadi M, Gácsi Z, Csarnovics I, et al. Enhanced growth of tellurium nanowires under conditions of macromolecular crowding. Phys Chem Chem Phys 2017;19:16477-84.

[141] Zhou G, Addou R, Wang Q, et al. High-mobility helical tellurium field-effect transistors enabled by transfer-free, low-temperature direct growth. Adv Mater 2018;30:1803109.

[142] Kang S, Dai T, Ma X, et al. Broad spectral response of an individual tellurium nanobelt grown by molecular beam epitaxy. Nanoscale 2019;11:1879-86.

[143] Basnet R, Doha MH, Hironaka T, et al. Growth and strain engineering of trigonal Te for topological quantum phases in non-symmorphic chiral crystals. Crystals 2019;9:486.

[144] Lin Y, Wu Y, Wang R, et al. Two-dimensional tellurium nanosheets for photoacoustic imaging-guided photodynamic therapy. Chem Commun 2018;54:8579-82.

[145] Xie Z, Xing C, Huang W, et al. Ultrathin 2D nonlayered tellurium nanosheets: facile liquid-phase exfoliation, characterization, and photoresponse with high performance and enhanced stability. Adv Funct Mater 2018;28:1705833.

[146] Wu L, Huang W, Wang Y, et al. 2D tellurium based high-performance all-optical nonlinear photonic devices. Adv Funct Mater 2019;29:1806346.

[147] Wang Y, Qiu G, Wang R, et al. Field-effect transistors made from solution-grown two-dimensional tellurene. Nat Electron 2018;1:228.

[148] Khatun S, Banerjee A, Pal AJ. Nonlayered tellurene as an elemental 2D topological insulator: experimental evidence from scanning tunneling spectroscopy. Nanoscale 2019;11:3591-8.

[149] Arora S, Vijay YK. Electrical, structural and optical properties of tellurium thin films on silicon substrate. AIP Conference Proceedings AIP Publishing 2018;1953:030083.

[150] Zhao C, Tan C, Lien DH, et al. Evaporated tellurium thin films for p-type field-effect transistors and circuits. Nat Nanotechnol 2020;15:53-8.

[151] Wu W, Qiu G, Wang Y, et al. Tellurene: its physical properties, scalable nanomanufacturing, and device applications. Chem Soc Rev 2018;47:7203-12.

[152] Anzin VB, Eremets MI, Kosichkin YV, et al. Measurement of the energy gap in tellurium under pressure. Phys Status Solidi A 1977;42:385-90.

[153] Pan Y, Gao S, Yang L, et al. Dependence of excited-state properties of tellurium on dimensionality: From bulk to two dimensions to one dimension. Phys Rev B 2018;98:085135.

[154] Qiao J, Pan Y, Yang F, et al. Few-layer tellurium: one-dimensional-like layered elementary semiconductor with striking physical properties. Sci Bull 2018;63:159-68.

[155] Niu C, Qiu G, Wang Y, et al. Gate-tunable strong spin-orbit interaction in two-dimensional tellurium probed by weakantilocalization. arXiv preprint arXiv:1909.06659, 2019.

[156] Nakayama K, Kuno M, Yamauchi K, et al. Band splitting and Weyl nodes in trigonal tellurium studied by angle-resolved photoemission spectroscopy and density functional theory. Phys Rev B 2017;95:125204.

[157] Huang W, Zhang Y, You Q, et al. Enhanced photodetection properties of tellurium@ selenium roll-to-roll nanotube heterojunctions. Small 2019;15:1900902. 
[158] Dang S, Kang S, Dai T, et al. Piezoelectric modulation of broadband photoresponse of flexible tellurium nanomesh photodetectors. Nanotechnology 2020;31:095502.

[159] Xie Z, Peng YP, Yu L, et al. Solar-inspired water purification based on emerging two-dimensional materials: status and challenges. Solar RRL 2020;1900400. https://doi.org/10.1002/ solr.201900400.

[160] Franklin AD. Nanomaterials in transistors: from high-performance to thin-film applications. Science 2015;349:aab2750.

[161] Wang Y, Qiu G, Wang Q, et al. Large-area solution-grown 2D tellurene for air-stable, high-performance field-effect transistors. arXiv preprint arXiv:1704.06202, 2017.

[162] Du Y, Qiu G, Wang Y, et al. One-dimensional van der Waals material tellurium: Raman spectroscopy under strain and magneto-transport. Nano Lett 2017;17:3965-73.

[163] Qin J, Qiu G, Jian J, et al. Controlled growth of a large-size 2D selenium nanosheet and its electronic and optoelectronic applications. ACS Nano 2017;11:10222-9.

[164] Abbaszadeh S, Allec N, Wang K, et al. Low dark-current lateral amorphous-selenium metal-semiconductor-metal photodetector. IEEE Electr Device L 2011;32:1263-5.

[165] Lee TI, Lee S, Lee E, et al. High-power density piezoelectric energy harvesting using radially strained ultrathin trigonal tellurium nanowire assembly. Adv Mater 2013;25:2920-5.

[166] Wang R, Su X, Bulla D, et al. Identifying the best chalcogenide glass compositions for the application in mid-infrared waveguides. Proc SPIE 2015;9444:944406.

[167] Xing C, Xie Z, Liang Z, et al. 2D nonlayered selenium nanosheets: facile synthesis, photoluminescence, and ultrafast photonics. Adv Opt Mater 2017;5:1700884.

[168] Fan T, Xie Z, Huang W, et al. Two-dimensional non-layered selenium nanoflakes: facile fabrications and applications for selfpowered photo-detector. Nanotechnology 2019;30:114002.

[169] Fu Q, Zhu C, Zhao X, et al. Ultrasensitive $2 \mathrm{D} \mathrm{Bi}_{2} \mathrm{O}_{2}$ Se phototransistors on silicon substrates. Adv Mater 2019;31:1804945.

[170] Wu J, Yuan H, Meng M, et al. High electron mobility and quantum oscillations in non-encapsulated ultrathin semiconducting $\mathrm{Bi}_{2} \mathrm{O}_{2}$ Se. Nat Nanotechnol 2017;12:530.

[171] Yin J, Tan Z, Hong H, et al. Ultrafast and highly sensitive infrared photodetectors based on two-dimensional oxyselenide crystals. Nat Commun 2018;9:3311.

[172] Wu J, Liu Y, Tan Z, et al. Chemical patterning of high-mobility semiconducting $2 \mathrm{D} \mathrm{Bi}_{2} \mathrm{O}_{2}$ Se crystals for integrated optoelectronic devices. Adv Mater 2017;29:1704060.

[173] Yang H, Tan C, Deng C, et al. Bolometric effect in $\mathrm{Bi}_{2} \mathrm{O}_{2} \mathrm{Se}$ photodetectors. Small 2019;15:e1904482.

[174] Wu Z, Liu G, Wang Y, et al. Seed-induced vertical growth of 2D $\mathrm{Bi}_{2} \mathrm{O}_{2} \mathrm{Se}$ nanoplates by chemical vapor transport. Adv Funct Mater 2019;29:1906639.

[175] Li J, Wang Z, Wen Y, et al. High-performance near-infrared photodetector based on ultrathin $\mathrm{Bi}_{2} \mathrm{O}_{2}$ Se nanosheets. Adv Funct Mater 2018;28:1706437.

[176] Meng M, Huang S, Tan C, et al. Strong spin-orbit interaction and magnetotransport in semiconductor $\mathrm{Bi}_{2} \mathrm{O}_{2}$ Se nanoplates. Nanoscale 2018;10:2704-10.

[177] Chen C, Wang M, Wu J, et al. Electronic structures and unusually robust bandgap in an ultrahigh-mobility layered oxide semiconductor, $\mathrm{Bi}_{2} \mathrm{O}_{2}$ Se. Sci Adv 2018;4: eaat8355.

[178] Tian X, Luo H, Wei R, et al. An ultrabroadband mid-infrared pulsed optical switch employing solution-processed bismuth oxyselenide. Adv Mater 2018;30:1801021.
[179] Zhang Z, Li T, Wu Y, et al. Truly concomitant and independently expressed short-and long-term plasticity in a $\mathrm{Bi}_{2} \mathrm{O}_{2}$ Se-based three-terminal memristor. Adv Mater 2019;31:1805769.

[180] Gao T, Zhang Q, Li L, et al. 2D ternary chalcogenides. Adv Opt Mater 2018;6:1800058.

[181] Fang J, Zhou Z, Xiao M, et al. Recent advances in low-dimensional semiconductor nanomaterials and their applications in high-performance photodetectors. InfoMat 2019;1-27. https://doi.org/10.1002/inf2.12067.

[182] Ganesha R, Arivuoli D, Ramasamy P. Growth of some group V-VI-VII compounds from the vapour. J Crys Growth 1993;128:1081-5.

[183] Zeng W, Li J, Feng L, et al. Synthesis of large-area atomically thin BiOl crystals with highly sensitive and controllable photodetection. Adv Funct Mater 2019;29:1900129.

[184] Zhao ZY, Dai WW. Electronic structure and optical properties of BiOl ultrathin films for photocatalytic water splitting. Inorg Chem 2015;54:10732-7.

[185] Hoye RLZ, Lee LC, Kurchin RC, et al. Strongly enhanced photovoltaic performance and defect physics of air-stable bismuth oxyiodide (BiOI). Adv Mater 2017;29:1702176.

[186] Ran Z, Wang X, Li Y, et al. Bismuth and antimony-based oxyhalides and chalcohalides as potential optoelectronic materials. NPJ Comput Mater 2018;4:14.

[187] Cui D, Wang L, Xu K, et al. Band-gap engineering of BiOCl with oxygen vacancies for efficient photooxidation properties under visible-light irradiation. J Mater Chem A 2018;6:2193-9.

[188] Kang L, Yu X, Zhao X, et al. Space-confined microwave synthesis of ternary-layered $\mathrm{BiOCl}$ crystals with high-performance ultraviolet photodetection. InfoMat 2019;1-8. https://doi. org/10.1002/inf2.12033.

[189] Ouyang W, Teng F, Fang X. High performance BiOCl nanosheets $/ \mathrm{TiO}_{2}$ nanotube arrays heterojunction UV photodetector: the influences of self-induced inner electric fields in the BiOCl nanosheets. Adv Funct Mater 2018;28:1707178.

[190] Jiang S, Li L, Wang Z, et al. Controlling magnetism in $2 \mathrm{D} \mathrm{Crl}_{3}$ by electrostatic doping. Nat Nanotechnol 2018;13:549-53.

[191] Huang B, Clark G, Klein DR, et al. Electrical control of 2D magnetism in bilayer $\mathrm{Crl}_{3}$. Nat Nanotechnol 2018;13:544-8.

[192] Song T, Cai X, Tu MWY, et al. Giant tunneling magnetoresistance in spin-filter van der Waals heterostructures. Science 2018;360:1214-8.

[193] Huang B, Clark G, Navarro-Moratalla E, et al. Layer-dependent ferromagnetism in a van der Waals crystal down to the monolayer limit. Nature 2017;546:270.

[194] Bonilla M, Kolekar S, Ma Y, et al. Strong room-temperature ferromagnetism in $\mathrm{VSe}_{2}$ monolayers on van der Waals substrates. Nat Nanotechnol 2018;13:289.

[195] Gong C, Li L, Li Z, et al. Discovery of intrinsic ferromagnetism in two-dimensional van der Waals crystals. Nature 2017;546:265.

[196] Wang Z, Zhang T, Ding M, et al. Electric-field control of magnetism in a few-layered van der Waals ferromagnetic semiconductor. Nat Nanotechnol 2018;13:554.

[197] Deng Y, Yu Y, Song Y, et al. Gate-tunable room-temperature ferromagnetism in two-dimensional $\mathrm{Fe}_{3} \mathrm{GeTe}_{2}$. Nature 2018;563:94.

[198] Yao Y, Zhang Y, Xiong W, et al. Growth and Raman scattering investigation of a new 2D MOX material: YbOCl. Adv Funct Mater 2019;29:1903017.

[199] Xie Z, Chen S, Duo Y, et al. Biocompatible two-dimensional titanium nanosheets for multimodal imaging guided cancer theranostics. ACS Appl Mater Interfaces 2019;11:22129-40. 
[200] Zhang Y, Lim CK, Dai Z, et al. Photonics and optoelectronics using nano-structured hybrid perovskite media and their optical cavities. Phys Rep 2019;795:1-51.

[201] Xu Q, Yang Z, Peng D, et al. $\mathrm{WS}_{2} / \mathrm{CsPbBr}_{3}$ van der Waals heterostructure planar photodetectors with ultrahigh on/ off ratio and piezo-phototronic effect-induced strain-gated characteristics. Nano Energy 2019;65:104001.

[202] Qin JK, Qiu G, He W, et al. Epitaxial growth of 1D atomic chain based Se nanoplates on monolayer $\mathrm{ReS}_{2}$ for high-performance photodetectors. Adv Funct Mater 2018;28:1806254. 